\title{
Supersymmetry and Dark Matter in Light of LHC 2010 and Xenon100 Data
}

\author{
O. Buchmueller ${ }^{\mathrm{a}}$, R. Cavanaugh ${ }^{\mathrm{b}, \mathrm{c}}$, D. Colling ${ }^{\mathrm{a}}$, A. De Roeck $^{\mathrm{d}, \mathrm{e}}$, M.J. Dolan ${ }^{\mathrm{f}}$, J.R. Ellis $^{\mathrm{d}, \mathrm{g}}$, \\ H. Flächer ${ }^{\mathrm{h}}$, S. Heinemeyer ${ }^{\mathrm{i}}$, G. Isidori ${ }^{\mathrm{j}}$, D. Martínez Santos ${ }^{\mathrm{d}}$, K.A. Olive ${ }^{\mathrm{k}}$, S. Rogerson ${ }^{\mathrm{a}}$, \\ F.J. Ronga ${ }^{\mathrm{l}}$, G. Weiglein ${ }^{\mathrm{m}}$
}

${ }^{\text {a} H i g h ~ E n e r g y ~ P h y s i c s ~ G r o u p, ~ B l a c k e t t ~ L a b o r a t o r y, ~ I m p e r i a l ~ C o l l e g e, ~ P r i n c e ~ C o n s o r t ~ R o a d, ~ L o n d o n ~ S W 7 ~ 2 A Z, ~ U K ~}$

${ }^{\mathrm{b}}$ Fermi National Accelerator Laboratory, P.O. Box 500, Batavia, Illinois 60510, USA

${ }^{c}$ Physics Department, University of Illinois at Chicago, Chicago, Illinois 60607-7059, USA

${ }^{\mathrm{d}}$ CERN, CH-1211 Genève 23, Switzerland

${ }^{\mathrm{e}}$ Antwerp University, B-2610 Wilrijk, Belgium

${ }^{\mathrm{f}}$ Institute for Particle Physics Phenomenology, University of Durham, South Road, Durham DH1 3LE, UK

'Theoretical Physics and Cosmology Group, Department of Physics, King's College London, London WC2R 2LS, UK

${ }^{\mathrm{h}}$ Department of Physics and Astronomy, University of Rochester, Rochester, New York 14627, USA

instituto de Física de Cantabria (CSIC-UC), E-39005 Santander, Spain

${ }^{j}$ INFN, Laboratori Nazionali di Frascati, Via E. Fermi 40, I-00044 Frascati, Italy

${ }^{k}$ William I. Fine Theoretical Physics Institute, University of Minnesota, Minneapolis, Minnesota 55455, USA

${ }^{1}$ Institute for Particle Physics, ETH Zürich, CH-8093 Zürich, Switzerland

${ }^{\mathrm{m}}$ DESY, Notkestrasse 85, D-22607 Hamburg, Germany

We make frequentist analyses of the CMSSM, NUHM1, VCMSSM and mSUGRA parameter spaces taking into account all the public results of searches for supersymmetry using data from the 2010 LHC run and the Xenon100 direct search for dark matter scattering. The LHC data set includes ATLAS and CMS searches for jets $+E_{T}$ events (with or without leptons) and for the heavier MSSM Higgs bosons, and the upper limit on $\mathrm{BR}\left(B_{s} \rightarrow \mu^{+} \mu^{-}\right)$including data from LHCb as well as CDF and D $\varnothing$. The absences of signals in the LHC data favour somewhat heavier mass spectra than in our previous analyses of the CMSSM, NUHM1 and VCMSSM, and somewhat smaller dark matter scattering cross sections, all close to or within the pre-LHC $68 \%$ CL ranges, but do not impact significantly the favoured regions of the mSUGRA parameter space. We also discuss the impact of the Xenon100 constraint on spin-independent dark matter scattering, stressing the importance of taking into account the uncertainty in the $\pi$-nucleon $\sigma$ term $\Sigma_{\pi N}$, that affects the spin-independent scattering matrix element, and we make predictions for spin-dependent dark matter scattering. Finally, we discuss briefly the potential impact of the updated predictions for sparticle masses in the CMSSM, NUHM1, VCMSSM and mSUGRA on future $e^{+} e^{-}$ colliders.

CERN-PH-TH/2011-129, DCPT/11/62, DESY 11-119, IPPP/11/31, FTPI-MINN-11/12, KCL-PH-TH/2011-12, LCTS/2011-01, UMN-TH-3002/11 


\section{Introduction}

One of the most appealing possible extensions of the Standard Model (SM) is supersymmetry (SUSY) 11. It would stabilize the electroweak mass hierarchy and facilitate grand unification, it predicts a relatively light Higgs boson that would be consistent with the indications from precision electroweak data, it offers a possible explanation of the apparent discrepancy between the experimental measurement of the anomalous magnetic moment of the muon, $(g-2)_{\mu}$, and the theoretical value calculated within the SM, and the lightest supersymmetric particle (LSP) is a plausible candidate for astrophysical dark matter.

We have published results from frequentist analyses of the minimal supersymmetric extension of the Standard Model (MSSM), using likelihood functions to take into account the experimental, phenomenological and astrophysical constraints on SUSY. The unconstrained MSSM contains too many parameters for a full exploration of its parameter space to be possible using present data, even including the current LHC data set of $\sim 35 / \mathrm{pb}$ [2]. Therefore, we have focused on making estimates within simplified versions of the MSSM, specifically the constrained MSSM (the CMSSM) [3,4] in which soft SUSY-breaking mass parameters are assumed to be universal at the GUT scale, in the simplest generalization of this model in which the universality is relaxed to allow non-universal Higgs masses (the NUHM1) [5]7, in a very constrained model in which the supplementary relation $A_{0}=B_{0}+m_{0}^{1}$ is imposed on trilinear and bilinear soft SUSY-breaking masses in the CMSSM (the VCMSSM) 8, 9, and in minimal supergravity (mSUGRA) in which, in addition, the gravitino mass $m_{3 / 2}$ is set equal to the common soft SUSY-breaking scalar mass $m_{0}$ before renormalization [8, 9. In each case, we assume that the LSP is the lightest neutralino $\tilde{\chi}_{1}^{0}$. More details on the model definitions can be found in 8 .

In a series of papers $[4,6,8$ ] we have presented predictions for Higgs and sparticle masses as well as for $\operatorname{BR}\left(B_{s} \rightarrow \mu^{+} \mu^{-}\right)$and the spin-independent

\footnotetext{
${ }^{1}$ We recall that our convention [2 8, for the sign of $A_{0}$ is opposite to that of SoftSUSY.
}

dark matter scattering cross section, $\sigma_{p}^{\text {SI }}$, and also for $m_{t}$ and $M_{W}$ [10. Most recently 2] we have included in global analyses the results of an initial CMS search in multijet $+\not_{T}$ channels (CMS $\left.\alpha_{T}\right)$ [1] and an ATLAS search in lepton + multijet $+\not_{T}$ channels (ATLAS 1L) 12$]^{2}$ Incorporating these new results led to upward shifts in the lower bounds on the gluino mass, $m_{\tilde{g}}$, by $\sim 100 \mathrm{GeV}$ in the models considered. Other masses connected to $m_{\tilde{g}}$, such as that of the lightest neutralino, $m_{\tilde{\chi}_{1}^{0}}$ (which we assume to provide the astrophysical cold dark matter (CDM) 14]) also moved upward by corresponding amounts. This in turn led to somewhat lower expectations for the spin-independent dark matter scattering cross section $\sigma_{p}^{\mathrm{SI}}$ in the models considered [2].

Subsequent to our analysis 2] of the implications of these initial LHC searches for SUSY, LHC experiments have provided several new constraints on SUSY using an integrated luminosity of $\sim 35 / \mathrm{pb}$ of data at $7 \mathrm{TeV}$. ATLAS has published the results of a search in multijet $+\mathbb{E}_{T}$ channels (ATLAS OL) 15] that has greater sensitivity in some regions to the types of gluino and squark pair-production events expected in the supersymmetric models discussed here than did the earlier ATLAS 1L search 12, and has also released results obtained by combining the one- and zero-lepton searches [16]. CMS has announced results from two other searches in multijet $+\mathbb{E}_{T}$ channels that improve the CMS $\alpha_{T}$ sensitivity also to gluino and squark production in the models discussed here. Both ATLAS and CMS have also published the results of searches for jets + $E_{T}$ events with $b$ tags [17, and for multilepton + jets $+E_{T}$ events [18. In addition, CMS and ATLAS have published new upper limits on the production of the heavier neutral MSSM Higgs bosons $H, A[19,20$, and LHCb has recently provided a new upper limit on $\operatorname{BR}\left(B_{s} \rightarrow \mu^{+} \mu^{-}\right)$[21], of comparable sensitivity to previous results from CDF [22] and D $\varnothing 23]$.

In parallel, the Xenon100 Collaboration has recently released results from a search for direct spin-independent dark matter scattering with

${ }^{2}$ Other analyses can be found in [13], where similar effects were found in the CMSSM and in gauge-mediated models. 
100.9 live days of data using a fiducial target with a mass of $48 \mathrm{~kg}$ [24. As we see later, this provides constraints on the parameter spaces of supersymmetric models that complement those provided by collider experiments 3 .

In this paper we combine these new constraints in updated global frequentist analyses of the parameter spaces of the CMSSM, NUHM1, VCMSSM and mSUGRA that take into account the results of all the searches using 2010 LHC data as well as the new Xenon100 constraint on the spin-independent scattering cross section, $\sigma_{p}^{\mathrm{SI}}$. At each point in the parameter spaces of these models, we construct a global likelihood function using previous data on electroweak precision observables, $(g-2)_{\mu}$ and $\mathrm{BR}(b \rightarrow s \gamma)$, and applying the strongest of the new constraints from searches for multijet $+E_{T}$ events, in combination with the constraints from $H / A$ searches, $\operatorname{BR}\left(B_{s} \rightarrow \mu^{+} \mu^{-}\right)$ and $\sigma_{p}^{\mathrm{SI}}$, via the implementations described in the next section.

The ATLAS and CMS searches for multijet + $E_{T}$ events provide constraints in complementary regions of the $\left(m_{0}, m_{1 / 2}\right)$ planes of these models, while the searches for heavier neutral MSSM Higgs bosons provide a relevant constraint in the $\left(M_{A}, \tan \beta\right)$ plane of the NUHM1. The LHCb search for $\operatorname{BR}\left(B_{s} \rightarrow \mu^{+} \mu^{-}\right)$, in combination with the CDF and $\mathrm{D} \varnothing$ searches, affects significantly the likelihood function for this observable, with particular relevance for the NUHM1. The best-fit points in our new fits including all these 2010 LHC constraints and the limit from the Xenon100 experiment are all close to or within the regions favoured by pre-LHC fits at the $68 \%$ CL. The spectra are somewhat heavier in the cases of the CMSSM, NUHM1 and VCMSSM, whereas the best-fit mSUGRA spectrum is little changed. The Xenon100 upper limit on $\sigma_{p}^{\mathrm{SI}}$ has little impact on the favoured regions of the VCMSSM and mSUGRA, and the impact on the

\footnotetext{
${ }^{3}$ See 25] for discussions of the Xenon100 results in the context of various models including the CMSSM. Ref. 26] compares LHC limits and the sensitivities of astrophysical searches for supersymmetric dark matter in specific CMSSM $\left(m_{0}, m_{1 / 2}\right)$ planes for fixed values of $\tan \beta$. Ref. 27 discusses the interplay between Xenon100 and LHC searches in the context of a no-scale flipped SU(5) model.
}

CMSSM and NUHM1 parameter spaces is limited by the present experimental uncertainty in the hadronic scattering matrix element, that is currently inherited primarily from the uncertainty in the low-energy $\pi$-N $\sigma$ term, $\Sigma_{\pi N}$. Based on the combination of 2010 LHC and Xenon100 constraints, we present updated likelihood functions for sparticle masses and other observables including $m_{\tilde{g}}, \operatorname{BR}\left(B_{s} \rightarrow \mu^{+} \mu^{-}\right)$and $\sigma_{p}^{\mathrm{SI}}$. We also present predictions for the spin-dependent scattering cross section, $\sigma_{p}^{\mathrm{SD}}$, that lie considerably below the present experimental upper limits. Finally, as an offshoot of our analysis, we discuss briefly the potential impact of our results on future $e^{+} e^{-}$colliders.

\section{Methodology}

Our analyses are performed using the MasterCode framework [2, 4, 6, 8, 10, 28]. The analyses have been made in a frequentist approach, in which we construct a global likelihood function with contributions from precision electroweak observables, $B$-physics observables, $(g-2)_{\mu}$ and the astrophysical cold dark matter density $\Omega_{\chi} h^{2}$ as well as the limits from the direct LEP searches for the Higgs boson and sparticles and, most recently, from sparticle searches at the LHC. The model parameter spaces are sampled using Markov Chain Monte Carlo (MCMC) techniques described in our previous papers. Our previous MCMC samplings of the CMSSM and NUHM1 parameter spaces each comprised some 25,000,000 points, whereas those of the VCMSSM and mSUGRA include some $30,000,000$ and 17,000,000 points, respectively. For the purposes of this paper we have added a sample of some 5,000,000 CMSSM points with $m_{0}<600 \mathrm{GeV}$ and $250 \mathrm{GeV}<m_{1 / 2}<800 \mathrm{GeV}$, designed to improve our understanding of the global likelihood function at values of $m_{1 / 2}$ that are somewhat larger than the previous best-fit values in our pre-LHC analysis of the CMSSM. This extra sampling had very little impact on our estimates of the best-fit points and 68 and $95 \%$ CL regions extracted from the $\chi^{2}$ evaluation, confirming the adequacy of our sampling in the parameter regions of interest. 
The pre-LHC constraints are also treated similarly to our previous analyses, see Ref. [2 for the most up-to-date description. The numerical evaluation within the MasterCode 2, 4, 6, 8, 10, 28, combines SoftSUSY [29] 4, FeynHiggs 30 33], SuFla 34, 35, SuperIso 36, 37, a code for electroweak observables based on [38, 39] and MicrOMEGAs 40] (with DarkSUSY 41] as an option not used in this paper), making extensive use of the SUSY Les Houches Accord [42,43]. The predictions we make for $\operatorname{BR}\left(B_{s} \rightarrow \mu^{+} \mu^{-}\right)$using MasterCode are checked for specific fit parameters using the independent SSARD code 44]. In the analysis of $\sigma_{p}^{\mathrm{SI}}$ in this paper, we link a part of SSARD to MasterCode to take account of hadronic uncertainties in dark matter scattering matrix elements, making cross-checks with MicrOMEGAs.

The MasterCode is designed in such a way that the constraints from new observables can be taken into account and incorporated quickly and easily into the global likelihood function as 'afterburners', i.e., by adding the calculated contribution to the likelihood function from the new observable and subsequently re-evaluating the global $\chi^{2}$ function. The new ingredients in this analysis coming from 2010 LHC and other searches are incorporated as just such 'afterburners', via the implementations described below.

\section{Implementations of $2010 \mathrm{LHC}$ and other Constraints}

Studies by the LHC Collaborations have shown that multijet $+\mathbb{E}_{T}$ constraints, with or without a single lepton, are relatively insensitive to $\tan \beta$ and $A_{0}$. Accordingly, we treat the ATLAS and CMS constraints on such signatures as independent of $\tan \beta$ and $A_{0}$, and regard their constraints in the $\left(m_{0}, m_{1 / 2}\right)$ plane as 'universal' [11, 12, 15, 45]. At each point in this plane, we compare the strengths of these ATLAS and CMS constraints, and retain the stronger, not attempting to combine the constraints from different experiments.

The constraints due to CMS and ATLAS

\footnotetext{
${ }^{4}$ In this paper we have upgraded from the version 2.0.11 used in earlier analyses to the new version 3.0.13: we indicate below where this change affects our analysis.
}

searches for events containing two or more leptons 18 are in general less sensitive than the constraints due to events with jet $+\not_{T}$ and at most one lepton, in the models considered here, and hence are not relevant for our evaluation of the global likelihood function. Moreover, these searches including leptons are also more sensitive to the value of $\tan \beta$, as are searches using $b$ tags [17. Since the reaches of the latter searches do not exceed those of the pure multijet $+\mathbb{F}_{T}$ searches, even at large $\tan \beta \sim 50$, they also do not contribute to the global likelihood function 5 .

\section{ATLAS jets $+\#_{T}+$ 0, 1 lepton analyses}

We treat the ATLAS analyses of events with multiple jets, zero or one lepton and $E_{T}$ (ATLAS 0L, ATLAS1L) 12, 15, 16 as follows. ATLAS reports the combined results of these searches as a $95 \% \mathrm{CL}$ exclusion contour in the $\left(m_{0}, m_{1 / 2}\right)$ plane for $\tan \beta=3$ and $A_{0}=06$. As seen in [16], the ATLAS 0L analysis provides the dominant constraint on $m_{1 / 2}$ for $m_{0}<300 \mathrm{GeV}$. Moreover, Fig. 17d of [46] shows that the ATLAS 0L search with the greatest impact on the parameter spaces of the CMSSM, NUHM1 and VCMSSM is ATLAS search D $\left(\geq 3\right.$ jets with leading $p_{T}>$ $120 \mathrm{GeV}$, other jets with $p_{T}>40 \mathrm{GeV}, \not_{T}>$ $100 \mathrm{GeV}, \Delta \phi\left(\right.$ jet,$\left.\not \phi_{T}\right)>0.4, m_{\mathrm{eff}}>1000 \mathrm{GeV}$, $\left.E_{T} / m_{\mathrm{eff}}>0.25\right)$.

Two events were observed in ATLAS 0L search $\mathrm{D}$, to be compared with the number of $2.5 \pm$ $1.0{ }_{-0.4}^{+1.0} \pm 0.2$ events expected due to SM backgrounds 7 We interpret this as a 'signal' of $-0.5 \pm$ 2.2 events, corresponding to a $95 \%$ CL upper limit of 3.8 events. This corresponds to the quoted $95 \%$ CL upper limit of $0.11 \mathrm{pb}$ and the $35 / \mathrm{pb}$ of integrated luminosity analyzed by ATLAS, and reproduces approximately the $95 \% \mathrm{CL}$ contour for search D shown in Fig. 17d of [46]. This figure

\footnotetext{
${ }^{5}$ We note in passing that LEP and Tevatron searches for sparticle pair-production also do not contribute significantly to the global likelihood function, whereas the LEP search for the lightest MSSM Higgs boson does contribute significantly.

${ }^{6}$ As mentioned above, this contour is not very sensitive to these choices: see the discussions in [12, 15, 16].

${ }^{7}$ These errors are due to the uncorrelated systematic uncertainty (including also the jet energy resolution and lepton efficiencies), the jet energy scale, and the luminosity, respectively.
} 
also reports the numbers of events expected in ATLAS search D for points with various different values of $\left(m_{0}, m_{1 / 2}\right)$. We calculate the corresponding numbers of effective deviations $\sigma_{\text {eff }}$ from the observed 'signal', and construct a map of the deviations for intermediate values of $\left(m_{0}, m_{1 / 2}\right)$ by interpolating between these values. At larger values of $\left(m_{0}, m_{1 / 2}\right)$, where expected event numbers are not provided, we scale the event numbers $\propto M^{-4}$, where $M \equiv \sqrt{m_{0}^{2}+m_{1 / 2}^{2}}$, following [2] and consistent with previous ATLAS studies. We then estimate the corresponding numbers of effective deviations $\sigma_{\text {eff }}$ from the observed 'signal' using the same prescription as above, and use this to calculate the corresponding value of $\chi^{2}$.

For $m_{0}>300 \mathrm{GeV}$, the best available ATLAS constraint on $m_{1 / 2}$ comes from a combination of the ATLAS 0L and ATLAS 1L analyses. To estimate the corresponding contribution to the likelihood function at larger $\left(m_{0}, m_{1 / 2}\right)$, we again use $M^{-4}$ scaling to estimate the expected numbers of events.

We evaluate the overall ATLAS contribution to $\chi^{2}$ for each of the points in our samples of the CMSSM, NUHM1, VCMSSM and mSUGRA parameter spaces by combining these treatments of the ATLAS searches at small and large $m_{0}$.

\section{CMS multijet $+E_{T}$ analyses}

Following the initial $\alpha_{T}$ analysis [11] that we analyzed previously [2, results from an additional CMS multijet $+\mathscr{E}_{T}$ analysis have been released (CMS MHT) 47] which has greater sensitivity in the $\left(m_{0}, m_{1 / 2}\right)$ plane. The CMS MHT analysis also imposes stronger constraints in the $\left(m_{0}, m_{1 / 2}\right)$ plane than does the ATLAS combined analysis [16] when $m_{0}>600 \mathrm{GeV}$, so we now analyze its results in more detail. The limit obtained in this search is very close to the median expected limit, corresponding to a difference between the numbers of events observed and expected from background that is negligible compared to the $\sigma_{\text {eff }}$ for the number of background events. We therefore approximate the impact of this search outside its nominal 95\% CL contour again by assuming that the number of effective $\sigma$ is simply proportional to the number of signal events expected at any given supersymmetric point, which we assume to be $\propto M^{-4}$, following [2], and we then calculate the corresponding $\chi^{2}$ penalty.

\section{Combining information of ATLAS and CMS analyses}

In our implementation of the combination of these constraints, for each supersymmetric point we compare the contributions to $\chi^{2}$ from the ATLAS and CMS MHT searches calculated as described above, and retain just the larger of the two $\chi^{2}$ penalties, dropping the contribution from the lesser constraint. This procedure is conservative, but any non-trivial combination of the constraints would require an understanding of the common systematic uncertainties that is currently unavailable, and would be justified only if the ATLAS and CMS collaborations provided additional information making possible more detailed modelling of their likelihood functions.

We note in passing that both CMS and ATLAS have published limits on simplified models based on the above searches. These limits are not directly applicable to the classes of supersymmetric models considered here since, for example, they consider cases in which $m_{\tilde{q}} \gg m_{\tilde{g}} \gg m_{\tilde{\chi}_{1}^{0}}$ and gluinos decay exclusively to $\bar{q} q \tilde{\chi}_{1}^{0}$, whereas in the models considered here other gluino decay modes are also important.

\section{$L H C$ searches for $H / A \rightarrow \tau^{+} \tau^{-}$}

The ATLAS and CMS Collaborations have also released the results of searches for heavier MSSM Higgs bosons $H / A$, produced mainly via $b \bar{b} \rightarrow H / A$ and decaying to $\tau^{+} \tau^{-}$pairs [19, 20]. The stronger of these constraints is provided by the CMS Collaboration, which we implement as follows. The CMS Collaboration has provided model-independent limits on the $H / A$ production cross section times $\tau^{+} \tau^{-}$branching ratio $(\sigma \times \mathrm{BR})$ at the $68 \%, 95 \%$ and $99.7 \%$ CLs as functions of $M_{A}$ [48, corresponding to a onedimensional $\chi^{2}$ contribution of $1,3.84$, and 9 , respectively. For each fixed value of $M_{A}$, we assume that the $\chi^{2}$ penalty for other values of $\sigma \times \mathrm{BR}$ may be approximated by the functional form $\Delta \chi^{2} \propto(\sigma \times \mathrm{BR})^{p\left(M_{A}\right)}$, normalized to unity on the $68 \% \mathrm{CL}$ line and fitting the power $p\left(M_{A}\right)$ 
independently for each value of $M_{A}$ (typical values are $\sim 1.3)$. The existing CMS bounds on $b \bar{b} \rightarrow$ $H / A \rightarrow \tau^{+} \tau^{-}$are expected to impact significantly only the NUHM1 scenario, where relatively low values of $M_{A}$ and high values of $\tan \beta$ lie within the region allowed by other constraints at the $95 \% \mathrm{CL}$. Therefore, we have evaluated $\sigma \times \mathrm{BR}$ for a representative grid of points in the NUHM1 by using the the SM result for $\sigma\left(b \bar{b} \rightarrow H_{\mathrm{SM}}\right)$ 49] modified by the effective NUHM1 couplings obtained from FeynHiggs, which we also use to calculate the branching ratio for the decay to $\tau^{+} \tau^{-}$. A factor of two is included to take into account the production of the $\mathrm{CP}$-even $H$ and the $\mathrm{CP}$ odd $A$ boson, which have approximately the same production cross section and decay widths in the relevant parameter space, $M_{A} \geq 150 \mathrm{GeV}$ and large $\tan \beta$. We have then checked that $\sigma \times \mathrm{BR}$ for fixed $M_{A}$ has a dependence $\sim \tan ^{2} \beta$ in the parameter regions of interest. Using the value of $\sigma \times \mathrm{BR}$ calculated in this way for each point in the NUHM1 parameter space, we then apply the $\chi^{2}$ penalty estimated as described above as an afterburner in our global fit.

$L H C b, C D F$ and DØ searches for $B_{s} \rightarrow \mu^{+} \mu^{-}$

The paper by $\mathrm{LHCb} 21$ provides $95 \%$ and $90 \%$ upper limits on $\operatorname{BR}\left(B_{s} \rightarrow \mu^{+} \mu^{-}\right)$of 56 and $43 \times 10^{-9}$, to be compared with the Standard Model prediction of $(3.2 \pm 0.2) \times 10^{-9}$. These limits are similar to the ones provided by CDF 22 . and $\mathrm{D} \varnothing$ [23], and a combination of the results from the three experiments provides a stronger constraint on $\operatorname{BR}\left(B_{s} \rightarrow \mu^{+} \mu^{-}\right)$. In order to make such a combination, we first performed approximate studies, based on the signal and background expectations in each experiment, and comparing with the observed pattern of events, generating toy experiments that reproduce their quoted 90\% CL upper limits. The toy LHCb experiment was constructed using the infomation shown in Table 3 of [21. The toy CDF experiment was based on the information given in Table II of 22, combined with the invariant mass resolution, normalization factors and averaged Neural Network efficiencies quoted in the text. In order to match exactly the observed $90 \%$ limit quoted by CDF, a small difference in the Neural Network efficiencies between the CMU-CMU and CMUCMX channels 22] was introduced. Finally, the toy DØ experiment was based on Fig. 4 of [23, together with the invariant mass resolution and normalization factor quoted in the text. These toy experiments also reproduce the quoted $95 \%$ CL limits, giving some support to this approximate treatment. The Tevatron results were afterwards recomputed using the latest world average $f_{d} / f_{s}=3.71 \pm 0.47[50$, for consistency with the LHCb analysis. The results of the three experiments were combined using the $C L_{s}$ method, treating the error on $f_{d} / f_{s}$ and the branching ratio of $B^{+} \rightarrow J / \psi\left(\mu^{+} \mu^{-}\right) K^{+}$as systematic errors common to the three experiments. The combined likelihood function yields formal upper limits of $20(24) \times 10^{-9}$ at the $90(95) \%$ CL: our global fit uses the full likelihood function calculated using the above experimental information to beyond the $99 \%$ CL.

\section{Xenon100 search for dark matter scattering}

Finally, we implement the constraint imposed by the direct upper limit on dark matter scattering given by the Xenon100 experiment 24]. Its results are presented as a 95\% CL upper limit on the spin-independent cross section as a function of $m_{\tilde{\chi}_{1}^{0}}$, under assumptions for the local halo density and the dark matter velocity distribution that are described in 24] and have uncertainties that are small compared to that in the spin-independent scattering matrix element discussed below 51,52. The Xenon100 Collaboration report the observation of 3 events in 100.9 live days within a fiducial detector with a mass of $48 \mathrm{~kg}$, in a range of recoil energies where $1.8 \pm 0.6$ events were expected 8 . Using this information, we have constructed a model for the Xenon100 contribution to the global $\chi^{2}$ likelihood function as a function of the number of events using the $C L_{s}$ method, which is quite similar to a Gaussian function with mean 1.2 and standard deviation 3.2 events. Our model for the Xenon100 likelihood function yields a 90\% CL upper limit of 6.1 events so, for any given value of $m_{\tilde{\chi}_{1}^{0}}$, we assume

\footnotetext{
${ }^{8}$ The probability for such a Poisson background process to yield 3 or more events is $28 \%$, so this observation does not constitute a significant signal.
} 
that the $90 \%$ CL upper limit on $\sigma_{p}^{\text {SI }}$ quoted in 24 corresponds to 6.1 events, and use simple scaling to estimate the event numbers corresponding to other values of $\sigma_{p}^{\mathrm{SI}}$. We then use the Gaussian model for the Xenon100 $\chi^{2}$ function to estimate the contribution of this experiment to the global likelihood function for other $\sigma_{p}^{\mathrm{SI}}$ values. We note that, because of the insignificant 'excess' of 1.2 events in the Xenon100 data, there is a contribution $\Delta \chi^{2} \sim 0.3$ to the global likelihood function at small values of $\sigma_{p}^{\mathrm{SI}} 9$.

In order to translate this estimate into contributions to the global likelihood functions for various supersymmetric models, we must take account of the uncertainty in the calculation of $\sigma_{p}^{\mathrm{SI}}$ for fixed supersymmetric model parameters. The dominant uncertainty is that in the determination of the strange quark scalar density in the nucleon, $\langle N|\bar{s} s| N\rangle$, which is induced principally by the experimental uncertainty in the $\pi$-nucleon $\sigma$ term, $\Sigma_{\pi N} \equiv 1 / 2\left(m_{u}+m_{d}\right)\langle N|\bar{u} u+\bar{d} d| N\rangle:$

$y \equiv \frac{2\langle N|\bar{s} s| N\rangle}{\langle N|\bar{u} u+\bar{d} d| N\rangle}=1-\frac{\sigma_{0}}{\Sigma_{\pi N}}$,

where $\sigma_{0} \equiv 1 / 2\left(m_{u}+m_{d}\right)\langle N|\bar{u} u+\bar{d} d-2 \bar{s} s| N\rangle=$ $36 \pm 7 \mathrm{MeV}[53$ is estimated from baryon octet mass splittings. Estimates of $\Sigma_{\pi N}$ ranging from $\sigma_{0}$ (corresponding to $y=0$ ) up to a value as large as $64 \pm 8 \mathrm{MeV}$ have been given in the literature 54 (and even larger values cannot be excluded [55]), whereas a recent analysis based on lattice calculations 56 would suggest a lower value: $\Sigma_{\pi N} \sim 40 \mathrm{MeV}$ [57. Here we span the plausible range by using as our default $\Sigma_{\pi N}=$ $50 \pm 14 \mathrm{MeV}$, while also showing some results for $\Sigma_{\pi N}=64 \pm 8 \mathrm{MeV} 10$.

The uncertainty in $\Sigma_{\pi N}$ is quite significant for our analysis, since it corresponds to an uncertainty in the spin-independent cross section for

\footnotetext{
${ }^{9}$ The predicted values of $\sigma_{p}^{\text {SI }}$ at the post-2010-LHC bestfit points are all smaller than preferred by this 'excess', so they all receive $\Delta \chi^{2} \sim 0.3$ from the Xenon100 data, as seen in the Table 1 For this reason, the lower $68 \%$ CL limits on $\sigma_{p}^{\text {SI }}$ are essentially unchanged when the Xenon100 data are incorporated in the fits.

${ }^{10}$ The estimated uncertainty in $\sigma_{0}=36 \pm 7 \mathrm{MeV}$ is also included in our analysis, as are the smaller uncertainties associated with the quark masses.
}

fixed supersymmetric model parameters of a factor of 5 or more. We plea again for an effort to reduce this uncertainty by a new campaign of experimental measurements and/or lattice QCD calculations.

\section{Impacts of the LHC and Xenon100 Con- straints}

$\left(m_{0}, m_{1 / 2}\right)$ planes

We display in Fig. 1 the $\left(m_{0}, m_{1 / 2}\right)$ planes for the CMSSM (upper left), NUHM1 (upper right), VCMSSM (lower left) and mSUGRA (lower right), driven by the ATLAS OL and CMS MHT constraints but also taking into account the other 2010 LHC constraints discussed above, as well as the Xenon100 constraint. In these and subsequent plots, we show in all panels best-fit points (in green), 68 and 95\% CL regions (red and blue lines, respectively). Our pre-LHC results, taken from [2], are displayed as 'snowflakes' and dotted lines, and our post-2010-LHC/Xenon100 results are displayed as full stars and solid lines 11.

Pre-LHC, the most important lower limits on $m_{1 / 2}$ in these models were indirect, being provided by the lower limit on $M_{h}$ from LEP, which had considerably greater impact in these models than did the direct sparticle searches at LEP and the Tevatron. In each of the CMSSM, the NUHM1 and the VCMSSM the direct 2010 LHC constraints push the best-fit values of $m_{1 / 2}$ to significantly higher values, as well as their 68 and 95\% CL ranges 12 , whereas the effect of Xenon100 is not visible in this projection of the model parameter spaces. Thus the direct 2010 LHC limits are constraining these models substantially more strongly than the LEP Higgs constraint.

This can be seen explicitly in the panels of Fig. 22 which compare the effects of the LEP Higgs and 2010 LHC constraints on the CMSSM. The upper left panel shows the best-fit point, $68 \%$ and $95 \%$ CL contours without applying either the LEP or the 2010 LHC constraints, and

${ }^{11}$ Our pre-LHC results differ slightly from those given in 2 as we use updated software including SoftSUSY 3.0.13.

${ }^{12} \mathrm{On}$ the other hand, the best-fit mSUGRA point is raised somewhat less, due to the different form of the global $\chi^{2}$ function. 

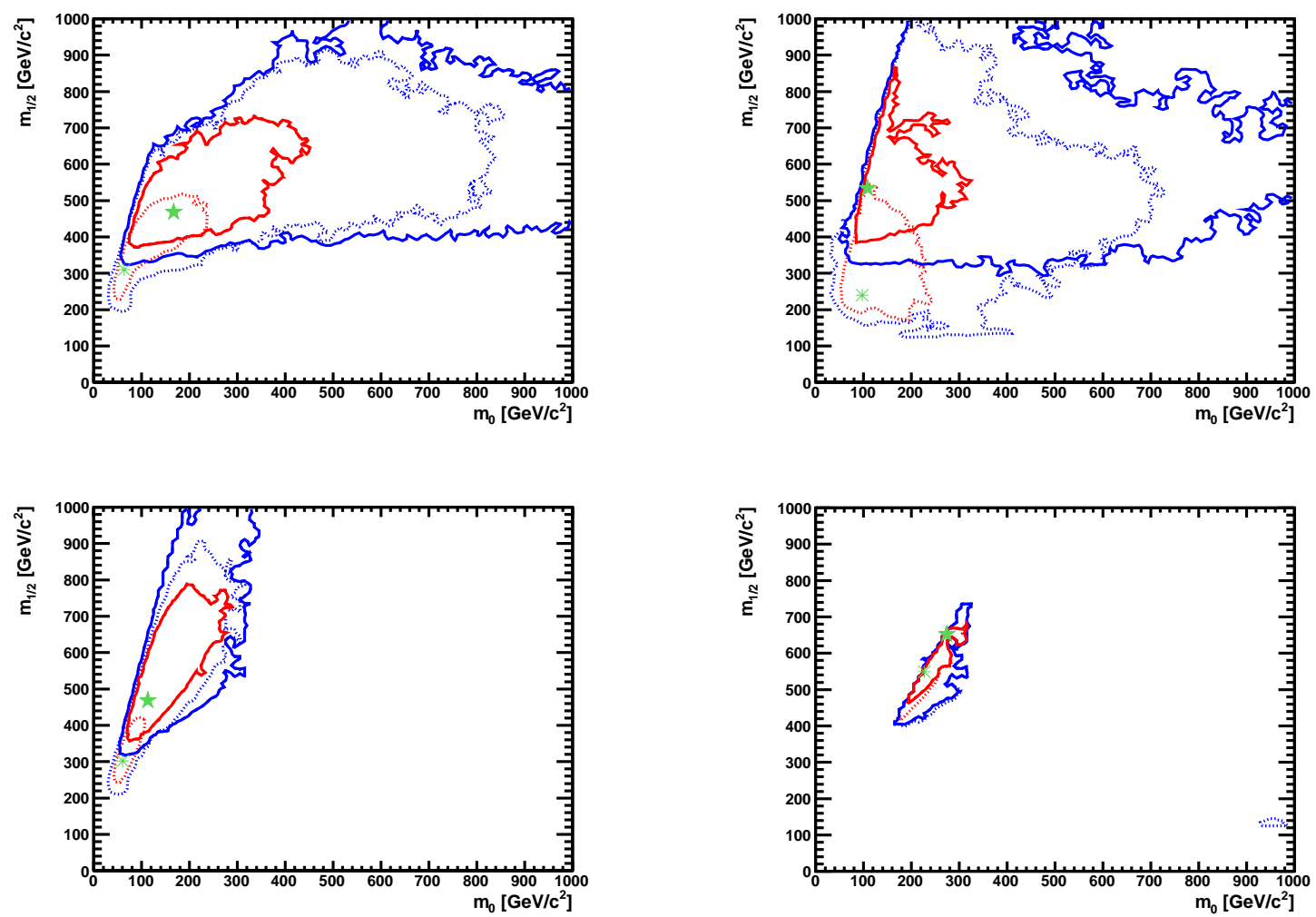

Figure 1. The $\left(m_{0}, m_{1 / 2}\right)$ planes in the CMSSM (upper left), the NUHM1 (upper right), the VCMSSM (lower left) and mSUGRA (lower right). In each plane, the best-fit point after incorporation of the 2010 LHC and Xenon100 constraints is indicated by a filled green star, and the pre-LHC fit by an open star. The 68 and 95\% CL regions are indicated by red and blue contours, respectively, the solid lines including the 2010 LHC and Xenon100 data, and the dotted lines showing the pre-LHC fits.

the upper right panel shows the effect of applying the LEP Higgs constraint but not the 2010 LHC constraints. We note that LEP moves the best-fit from $m_{1 / 2} \sim 270 \mathrm{GeV}$ to $\sim 320 \mathrm{GeV}$ while the $95 \%$ CL contour at large $m_{0}$ and $m_{1 / 2}$ expands slightly, reflecting the small rise in the minimum of $\chi^{2}$. The lower left panel shows the best-fit point, $68 \%$ and $95 \%$ CL contours applying the 2010 LHC constraints without the LEP Higgs constraint. The best-fit point now moves to $m_{1 / 2} \sim 470 \mathrm{GeV}$, and the $95 \% \mathrm{CL}$ contour moves correspondingly much further out. Finally, the lower right panel shows the effect of applying the LEP Higgs constraint as well. We see that the best fit remains essentially unchanged at $m_{1 / 2} \sim 470 \mathrm{GeV}$, and the $95 \% \mathrm{CL}$ contour is little affected at large $m_{0}$ and $m_{1 / 2}$. In summary, applying the LEP Higgs constraint increases $m_{1 / 2}$ by $\sim 50 \mathrm{GeV}$ in the absence of the LHC constraints, and only marginally if they are applied, whereas the LHC constraints increase $m_{1 / 2}$ by $\sim 200 \mathrm{GeV}$ in the absence of the LEP Higgs constraint, and by $\sim 150 \mathrm{GeV}$ if it is applied. Correspondingly, the effects of LHC on the 95\% CL contour are much greater than those of the LEP Higgs constraint.

As seen in Fig. 1. the effects of the LHC on the best-fit values of $m_{0}$ are smaller, though 

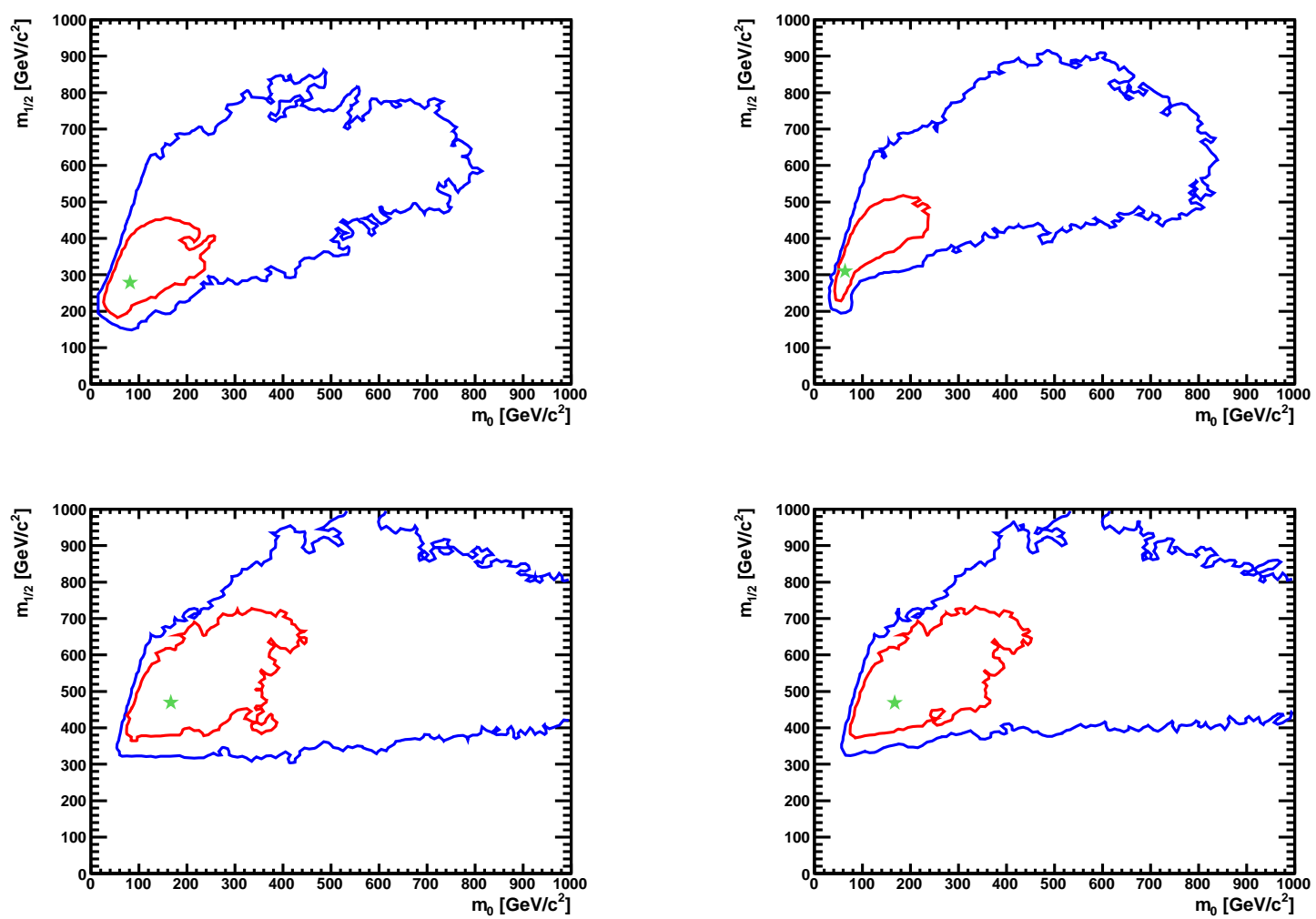

Figure 2. The $\left(m_{0}, m_{1 / 2}\right)$ planes in the CMSSM with neither the LEP Higgs constraints nor the LHC constraints applied (upper left), with LEP but without the LHC (upper right), without LEP but with the $L H C$ (lower left) and with both LEP and the LHC (lower right). In each plane, the best-fit point is indicated by a filled green star, and the 68 and 95\% CL regions are indicated by red and blue contours, respectively.

there are significant increases in the CMSSM and VCMSSM that are correlated with the increases in $m_{1 / 2}$. We note that in all models the new bestfit point lies within or close to the border of the pre-LHC $68 \%$ CL contour, indicating that there is no significant tension between the LHC constraints and prior indications on the scale of supersymmetry breaking. Nevertheless, in all cases other than mSUGRA, the pre-LHC best fit points are now excluded at the 95\% CL. Furthermore. the 2010 LHC constraints exclude roughly half of the pre-LHC $68 \%$ CL regions in the CMSSM and VCMSSM, and most of the pre-LHC 68\% CL region in the NUHM1. However, the LHC has yet to make any significant inroad into even the $95 \%$ CL region of mSUGRA 13 .

In Table 1 we compare the post-2010LHC/Xenon100 best-fit points found in this paper with pre-LHC results 2 in the CMSSM, NUHM1, VCMSSM and mSUGRA (in the latter case, only the best fit in the coannihilation region is reported). In addition to the minimum value of $\chi^{2}$, the number of degrees of freedom, and the fit

\footnotetext{
${ }^{13}$ The raggedness of the CL contours should be regarded as indicative of the uncertainties in our analysis. Recall also that, as already mentioned, our pre-LHC results differ slightly from those given in 2], as updated software was used, in particular SoftSUSY 3.0.13.
} 


\begin{tabular}{|c||c|c|c|c|c|c||c|}
\hline Model & $\begin{array}{c}\text { Minimum } \\
\chi^{2} / \text { dof }\end{array}$ & Probability & $\begin{array}{c}m_{1 / 2} \\
(\mathrm{GeV})\end{array}$ & $\begin{array}{c}m_{0} \\
(\mathrm{GeV})\end{array}$ & $\begin{array}{c}A_{0} \\
(\mathrm{GeV})\end{array}$ & $\tan \beta$ & $\begin{array}{c}M_{h}(\mathrm{GeV}) \\
(\mathrm{no} \mathrm{LEP})\end{array}$ \\
\hline \hline CMSSM pre-LHC & $22.5 / 19$ & $26 \%$ & $310_{-50}^{+120}$ & $60_{-10}^{+90}$ & $-60_{-840}^{+410}$ & $10_{-4}^{+10}$ & 108.6 \\
post-2010-LHC & $26.1 / 19$ & $13 \%$ & $470_{-70}^{+140}$ & $170_{-80}^{+330}$ & $-780_{-820}^{+1410}$ & $22_{-13}^{+27}$ & 115.7 \\
post-Xenon (50 \pm 14$)$ & $26.2 / 20$ & $16 \%$ & $470_{-70}^{+140}$ & $170_{-80}^{+330}$ & $-780_{-820}^{+1410}$ & $22_{-13}^{+27}$ & 115.7 \\
\hline NUHM1 pre-LHC & $20.5 / 17$ & $25 \%$ & $240_{-50}^{+150}$ & $100_{-40}^{+70}$ & $920_{-120}^{+360}$ & $7_{-2}^{+11}$ & 119.4 \\
post-2010-LHC & $24.1 / 18$ & $15 \%$ & $530_{-90}^{+220}$ & $110_{-20}^{+80}$ & $-370_{-1000}^{+1070}$ & $27_{-10}^{+24}$ & 117.9 \\
post-Xenon (50 \pm 14$)$ & $24.2 / 19$ & $19 \%$ & $530_{-90}^{+220}$ & $110_{-20}^{+80}$ & $-370_{-100}^{+1070}$ & $27_{-10}^{+24}$ & 117.9 \\
\hline VCMSSM pre-LHC & $22.6 / 20$ & $31 \%$ & $300_{-40}^{+60}$ & $60_{-10}^{+20}$ & $30_{-30}^{+50}$ & $8_{-1}^{+3}$ & 110.0 \\
post-2010-LHC & $27.9 / 20$ & $11 \%$ & $470_{-80}^{+150}$ & $110_{-30}^{+110}$ & $120_{-190}^{+300}$ & $13_{-8}^{+14}$ & 115.0 \\
post-Xenon (50 \pm 14$)$ & $28.1 / 21$ & $14 \%$ & $470_{-80}^{+150}$ & $110_{-30}^{+110}$ & $120_{-190}^{+300}$ & $13_{-8}^{+14}$ & 115.0 \\
\hline mSUGRA pre-LHC & $29.4 / 19$ & $6.0 \%$ & $550_{-90}^{+170}$ & $230_{-40}^{+80}$ & $430_{-90}^{+190}$ & $28_{-2}^{+5}$ & 107.8 \\
post-2010-LHC & $30.2 / 20$ & $6.7 \%$ & $650_{-130}^{+70}$ & $270_{-50}^{+50}$ & $530_{-130}^{+130}$ & $30_{-3}^{+4}$ & 122.2 \\
post-Xenon (50 \pm 14$)$ & $30.3 / 21$ & $8.6 \%$ & $650_{-130}^{+70}$ & $270_{-50}^{+50}$ & $530_{-130}^{+130}$ & $30_{-3}^{+4}$ & 122.2 \\
\hline
\end{tabular}

Table 1

Comparison of the best-fit points found in the pre-LHC analysis in the CMSSM, the NUHM1, the VCMSSM and the coannihilation region of $m S U G R A$ [2, 6] 8], and our latest results incorporating the $C M S, A T L A S, L H C b, C D F, D \varnothing$ and Xenon100 constraints. We also include the minimum value of $\chi^{2}$ and the fit probability in each scenario, as well as the predictions for $M_{h}$ without imposing the LEP constraint.

probability in each scenario, we include the values of $m_{1 / 2}, m_{0}, A_{0}$ and $\tan \beta$ at all the best-fit points, as well as the respective one-dimensional $68 \%$ CL ranges, and the predictions for $M_{h}$ if the LEP Higgs constraint is neglected. We note again that the 2010 LHC constraints are significantly stronger than those from previous sparticle searches and the LEP Higgs limit, resulting in significant increases in the best-fit values of $m_{1 / 2}$ and smaller increases in $m_{0}$ in the CMSSM, NUHM1 and VCMSSM. We note also significant increases in the best-fit values of $\tan \beta$ in these models, which are required by the $(g-2)_{\mu}$ constraint in order to compensate for the larger values of $m_{1 / 2}$ and $m_{0}$. In the case of the VCMSSM, the scope for increasing $\tan \beta$ is restricted by the condition that $A_{0}=B_{0}+m_{0}$, which is largely responsible for the relatively large increase in $\chi^{2}$ post-2010-LHC14 The values of $A_{0}$ are poorly constrained in all the models, and we have

${ }^{14}$ We recall that our convention 20,8 for the sign of $A_{0}$ is opposite to that of SoftSUSY. checked that there is not a strong dependence of the $\chi^{2}$ of the NUHM1 on the non-universality between the soft supersymmetry-breaking contributions to the Higgs and sfermion masses, though small values of the former are somewhat preferred. We see that the minimum values of $\chi^{2}$ have been increased by the inclusion of the 2010 LHC data, in particular. These increases result in some decreases in the overall probabilities, though insufficient to call the models into question. The Xenon100 constraint causes only small changes in the best-fit parameters of the models studied, as well as small increases in the $\chi^{2}$ values and a corresponding small increase in the probability.

Since the constraint that most disfavours large supersymmetry-breaking masses is $(g-2)_{\mu}$, and since it is the interplay between this and the advancing LHC constraints that pushes the best fits towards larger values of $\tan \beta$, we have investigated the effect of dropping this constraint altogether. This possibility was explored previ- 
ously using the pre-LHC data set in [7, where it was found that the large- $m_{0}$ focus-point region was slightly disfavoured in the CMSSM and NUHM1, even when dropping the $(g-2)_{\mu}$ constraint, by a combination of other observables including $M_{W}$, in particular. Now, when $(g-2)_{\mu}$ is dropped, using the 2010 LHC data set (whether the Xenon100 constraint is included, or not) we find a secondary minimum in the focus-point region that is disfavoured in the CMSSM by $\Delta \chi^{2} \sim 1.0$, whereas this region was disfavoured by $\Delta \chi^{2} \sim 1.6$ when $(g-2)_{\mu}$ was dropped from the pre-LHC data set. In the case of the NUHM1, we do not find a clear secondary minimum in the focus-point region when $(g-2)_{\mu}$ is dropped post2010-LHC.

$\left(\tan \beta, m_{1 / 2}\right)$ planes

In Fig. 3 we display the $\left(\tan \beta, m_{1 / 2}\right)$ planes for the CMSSM (upper left), NUHM1 (upper right), VCMSSM (lower left) and mSUGRA (lower right). We see again that the best-fit point and likelihood contours in mSUGRA are only mildly affected by the LHC data, whereas there are significant increases in the best-fit values of $\tan \beta$ in the CMSSM, NUHM1 and VCMSSM that are correlated with the increases in $m_{1 / 2}$. As already commented in 2, these increases may be understood from the interplay of the LHC and $(g-2)_{\mu}$ constraints. It is well known that for fixed $\tan \beta(g-2)_{\mu}$ favours an elliptical band in the $\left(m_{0}, m_{1 / 2}\right)$ plane that moves to larger mass values as $\tan \beta$ increases. Hence the pressure of the LHC towards larger values of $m_{1 / 2}$ is reconciled with $(g-2)_{\mu}$ by increasing $\tan \beta$. It is apparent from the upper panels and the $68 \%$ CL ranges given in the Table that the constraints on the possible values of $\tan \beta$ in the CMSSM and NUHM1 were quite weak pre-LHC, and are still not very strong. In the lower left panel, we see that in the VCMSSM the best-fit value of $\tan \beta$ has increased and its range has broadened considerably post-2010-LHC 15 .

\footnotetext{
${ }^{15}$ Many early LHC analyses assumed $\tan \beta=3$ as a default. It is apparent from Fig. 3 that such low values were disfavoured even pre-LHC, and that a more plausible default choice post-2010-LHC would be $\tan \beta=10$ or more.
}

$\left(M_{A}, \tan \beta\right)$ planes

We display in Fig. 4 the corresponding bestfit points and 68 and $95 \%$ CL regions in the $\left(M_{A}, \tan \beta\right)$ planes for the CMSSM, NUHM1, VCMSSM and mSUGRA including the 2010 LHC and Xenon100 constraints. The LHC $b \bar{b} \rightarrow$ $H / A \rightarrow \tau^{+} \tau^{-}$constraint has some impact in the NUHM1, where a small part of the upper left region of the NUHM1 $\left(M_{A}, \tan \beta\right)$ plane has been disfavoured by this new constraint, whereas the previous Tevatron constraints on $H / A$ production had not impacted significantly the parameter spaces of any of the models.

Fig. 5 illustrates the effects of the CMS $H / A$ constraint and the $\mathrm{LHCb} / \mathrm{CDF} / \mathrm{D} \varnothing \mathrm{BR}\left(B_{s} \rightarrow\right.$ $\left.\mu^{+} \mu^{-}\right)$constraint on the $\left(M_{A}, \tan \beta\right)$ plane in the NUHM1. The other LHC constraints are applied in all panels, but not the Xenon100 constraint. The left panel drops both the $H / A \rightarrow \tau^{+} \tau^{-}$and $\mathrm{BR}\left(B_{s} \rightarrow \mu^{+} \mu^{-}\right)$constraints, and the right panel includes both constraints, and we note two principal effects. One is a contraction in the $68 \%$ CL region at lower $M_{A}$, resulting in the $68 \% \mathrm{CL}$ lower limit on $M_{A}$ increasing from $\sim 150 \mathrm{GeV}$ to $\sim 200 \mathrm{GeV}$, which is due to the $H / A$ constraint. The other effect is some erosion of the $68 \%$ CL region at large $\tan \beta>50$, reducing the upper limit on $M_{A}$ from $\sim 600 \mathrm{GeV}$ to $\sim 550 \mathrm{GeV}$, which is due to the $\operatorname{BR}\left(B_{s} \rightarrow \mu^{+} \mu^{-}\right)$constraint. However, we observe that the location of the best-fit point at $\left(M_{A}, \tan \beta\right) \sim(400 \mathrm{GeV}, 26)$ is quite insensitive to these constraints, indicating that they are not yet attacking the 'heartland' of the NUHM1 parameter space.

\section{$\left(A_{0} / m_{0}, \tan \beta\right)$ planes}

Fig. [6] displays the $\left(A_{0} / m_{0}, \tan \beta\right)$ planes in the CMSSM (upper left panel), in the NUHM1 (upper right panel), in the VCMSSM (lower left panel) and in mSUGRA (lower right panel). We see that the effect of the 2010 LHC constraints in the CMSSM is to push the preferred region towards negative values of $A_{0} / m_{0}$, largely as a result of the push towards larger values of $\tan \beta$ required to reconcile the LHC data with $(g-2)_{\mu}$. The effects of the available constraints in this plane are weaker in the NUHM1, particularly for larger values of $\tan \beta$. In the cases of 

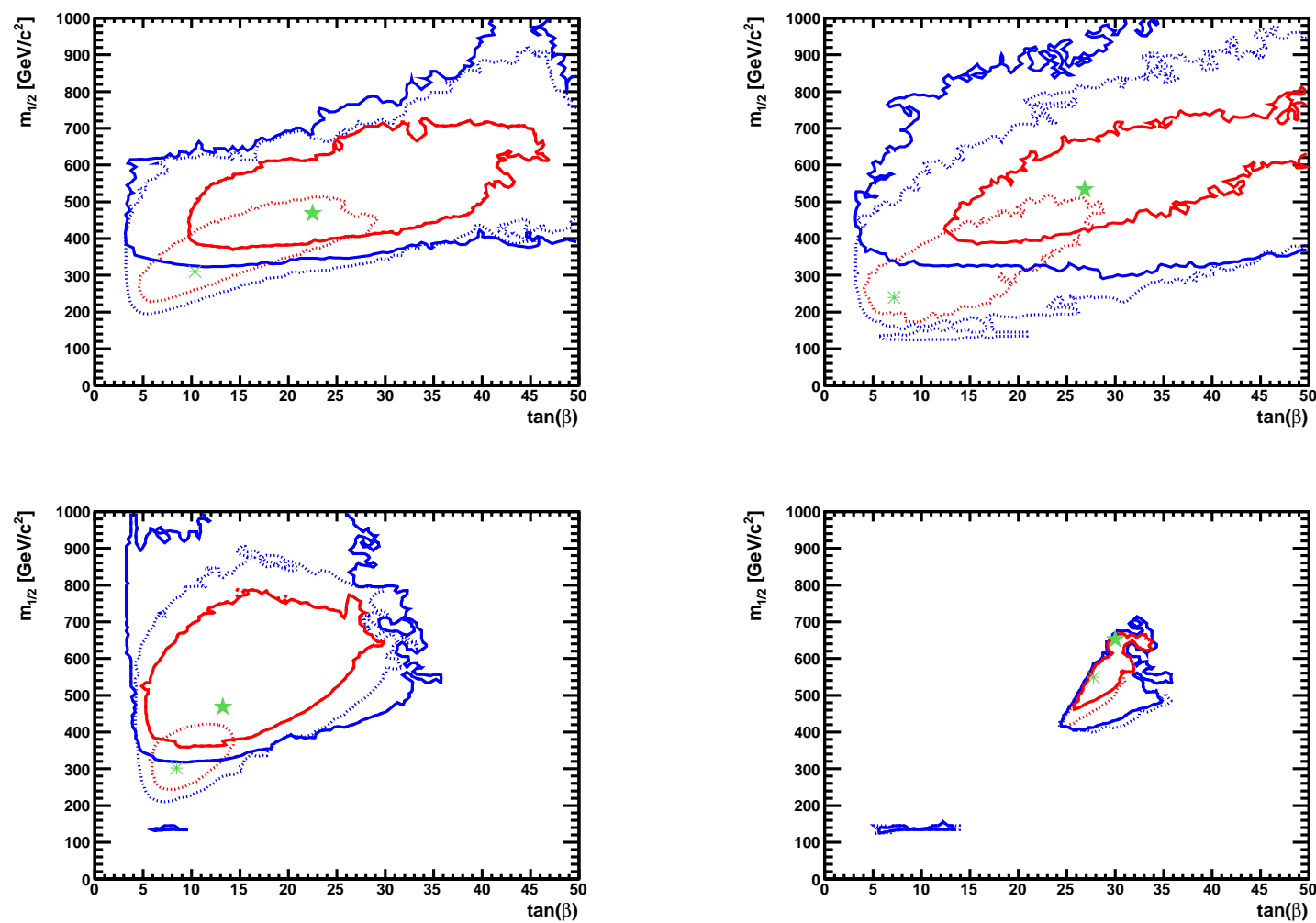

Figure 3. The $\left(\tan \beta, m_{1 / 2}\right)$ planes in the CMSSM (upper left), the NUHM1 (upper right), the VCMSSM (lower left) and mSUGRA (lower right). In each plane, the best-fit point after incorporation of the 2010 LHC and Xenon100 constraints is indicated by a filled green star, and the pre-LHC fit by an open star. The 68 and 95\% CL regions are indicated by red and blue contours, respectively, the solid lines including the 2010 LHC and Xenon100 data, and the dotted lines including only the pre-LHC data.

the VCMSSM and mSUGRA, we see that the $\left(A_{0} / m_{0}, \tan \beta\right)$ planes are qualitatively similar to those shown in 8], the main difference being a shift of the best-fit point to larger $A_{0} / m_{0}$ and $\tan \beta$.

\section{Gluino mass}

Fig. 7 illustrates the impacts of the 2010 LHC data on the $\chi^{2}$ likelihood functions for $m_{\tilde{g}}$ in the different models. The plots display the $\Delta \chi^{2}$ contributions of the different fits relative to the respective best-fit points. The pre-LHC likelihood functions are shown as dotted lines, and the post-2010-LHC likelihood functions as solid lines. In each of the CMSSM, NUHM1 and VCMSSM, the general effect of the 2010 LHC data is to increase the preferred value of $m_{\tilde{g}}$ by $\sim 300 \mathrm{GeV}$ beyond our pre-LHC analyses 8, reaching $\sim 1000-1300 \mathrm{GeV}$, which is also some $100 \mathrm{GeV}$ beyond the results of our previous analyses using the initial CMS $\alpha_{T}$ and ATLAS 1L searches [2], whereas there is no significant effect on the likelihood function for $m_{\tilde{g}}$ in mSUGRA. Since the plots display the relative $\Delta \chi^{2}$ contributions, the differences in the overall $\chi^{2}$ between the pre- and post-2010-LHC minima of $\sim 4(6)$ in the CMSSM/NUHM1 (VCMSSM) are responsible for the differences between the pre- and post-2010- 

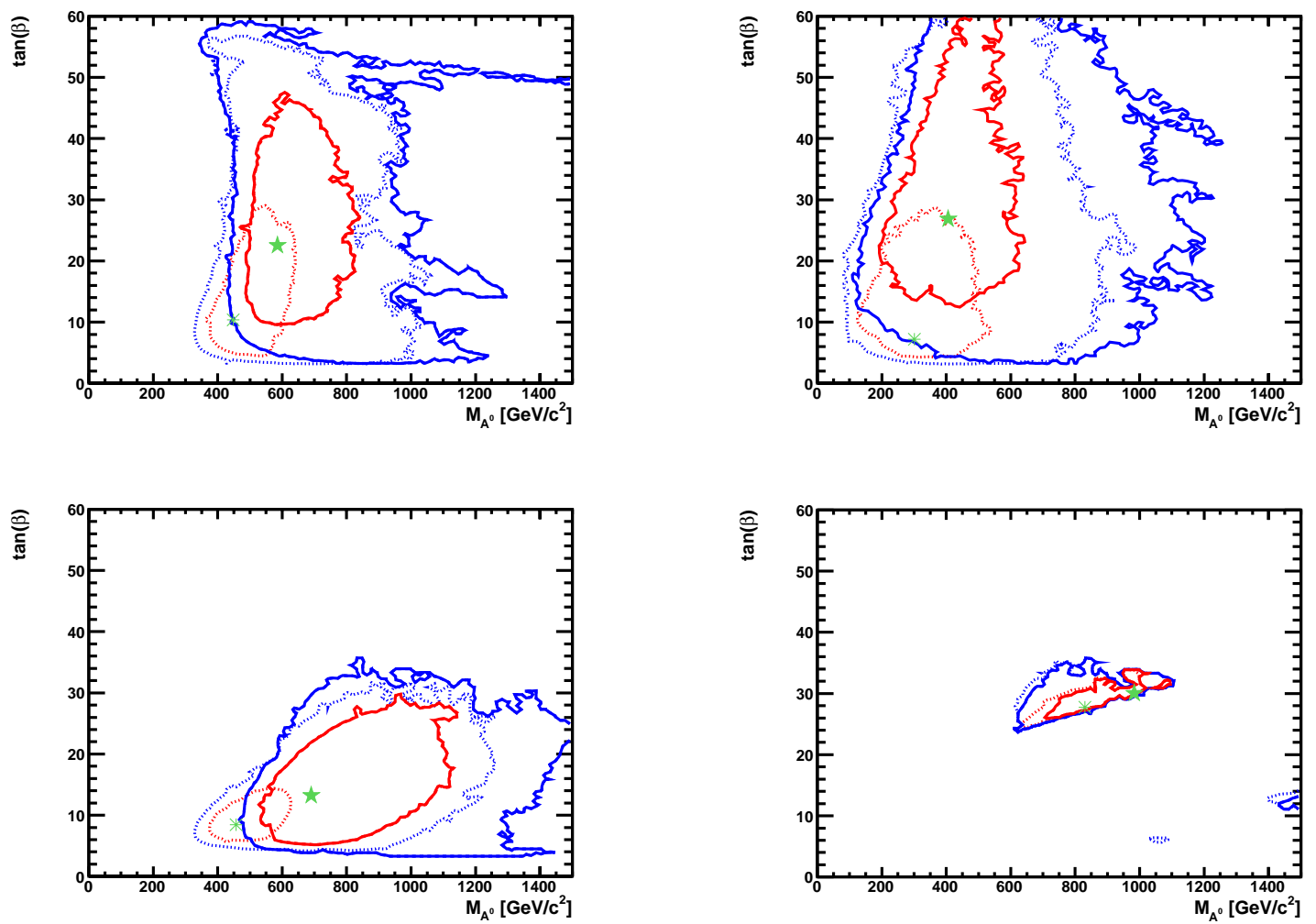

Figure 4. The $\left(M_{A}, \tan \beta\right)$ planes in the CMSSM (upper left), the NUHM1 (upper right), the VCMSSM (lower left) and mSUGRA (lower right). In each plane, the best-fit point after incorporation of the 2010 $L H C$ constraints is indicated by a filled green star, and the pre-LHC fit by an open star. The 68 and 95\% $C L$ regions are indicated by red and blue contours, respectively, the solid lines including the 2010 LHC data, and the dotted lines including only the pre-LHC data.

LHC likelihood functions at large $m_{\tilde{g}}$, where the LHC constraints have no effect on the absolute values of the $\chi^{2}$ functions. These new normalizations of $\chi^{2}$ are also responsible for the appearances of high-lying secondary minima at $m_{\tilde{g}} \sim$ $400 \mathrm{GeV}$ in the CMSSM and VCMSSM, respectively, which was previously located out of sight at $\Delta \chi^{2}>9$ for the CMSSM but has now dropped into view. These secondary minima, like that for mSUGRA, are compatible with the astrophysical cold dark matter density constraint thanks to rapid annihilation through a direct-channel light $h$ pole. The primary minima are located in the $\tilde{\tau}_{1}-\tilde{\chi}_{1}^{0}$ coannihilation regions, whereas the focus- point regions are strongly disfavoured in our analysis, and not seen in any panel of Fig. 7 .

\section{$\mathrm{BR}\left(B_{s} \rightarrow \mu^{+} \mu^{-}\right)$}

Fig. 8 displays the post-2010-LHC likelihood functions for $\operatorname{BR}\left(B_{s} \rightarrow \mu^{+} \mu^{-}\right)$, normalized to the SM prediction, where we see two principal effects. In the CMSSM and, to some extent, also in the VCMSSM (upper and lower left, respectively), values of $\operatorname{BR}\left(B_{s} \rightarrow \mu^{+} \mu^{-}\right)$exceeding the SM prediction are less disfavoured than in the pre-LHC case. This effect has a twofold origin. On the one hand, the LHC data disfavour a region of parameter space where a negative inter- 

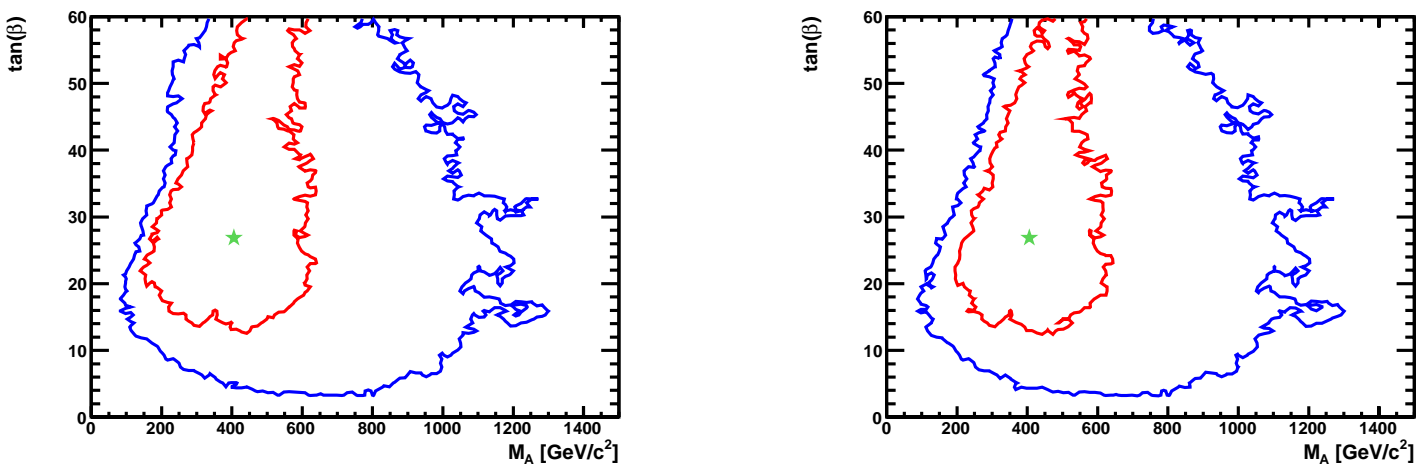

Figure 5. The $\left(M_{A}, \tan \beta\right)$ planes in the NUHM1 with neither the $C M S H / A \rightarrow \tau^{+} \tau^{-}$constraint nor the $\operatorname{BR}\left(B_{s} \rightarrow \mu^{+} \mu^{-}\right)$constraint applied (left) and with both the $H / A$ and the $\operatorname{BR}\left(B_{s} \rightarrow \mu^{+} \mu^{-}\right)$constraints (right). In each plane, the best-fit point is indicated by a filled green star, and the 68 and $95 \%$ CL regions are indicated by red and blue contours, respectively.

ference between SM and non-SM amplitudes gave rise to $\operatorname{BR}\left(B_{s} \rightarrow \mu^{+} \mu^{-}\right)$slightly below the $\mathrm{SM}$ prediction. On the other hand, the LHC data increase the minimum of $\chi^{2}$ significantly, and in this figure we show the $\Delta \chi^{2}$ contribution relative to the respective best-fit point. Since the absolute values of $\chi^{2}$ at large $\operatorname{BR}\left(B_{s} \rightarrow \mu^{+} \mu^{-}\right)$are essentially unchanged by the LHC data, the difference between the values of $\chi^{2}$ at the minimum and at large $\operatorname{BR}\left(B_{s} \rightarrow \mu^{+} \mu^{-}\right)$are also reduced by $\sim 4(6)$ in the CMSSM/NUHM1 (VCMSSM). In the NUHM1 case we observe another important effect: here values of $\operatorname{BR}\left(B_{s} \rightarrow \mu^{+} \mu^{-}\right)$much greater than the SM value (by a factor more than about 6) are now more disfavoured than in our previous analysis. This is due to the implementation of the $\mathrm{LHCb}, \mathrm{CDF}$ and $\mathrm{D} \varnothing$ constraints on $\mathrm{BR}\left(B_{s} \rightarrow \mu^{+} \mu^{-}\right)$that increases substantially the $\chi^{2}$ values at large $\operatorname{BR}\left(B_{s} \rightarrow \mu^{+} \mu^{-}\right)$. Nevertheless, we stress that a value of $\operatorname{BR}\left(B_{s} \rightarrow \mu^{+} \mu^{-}\right)$ that is substantially larger than the SM value is still more likely in the NUHM1 than in the other models (note the different horizontal scale used for the NUHM1).

\section{Light Higgs mass predictions}

In Fig. 9 the one-parameter $\chi^{2}$ functions for the lightest MSSM Higgs mass $M_{h}$ in the CMSSM,
NUHM1, VCMSSM and mSUGRA are shown. In this figure we do not include the direct limits from LEP [58, 59] or the Tevatron, so as to illustrate whether there is a conflict between these limits and the predictions of supersymmetric models. For each model we display the new likelihood functions corresponding to the post-2010LHC data set, indicating the theoretical uncertainty in the calculation of $M_{h}$ of $\sim 1.5 \mathrm{GeV}$ by red bands. We also show, as dashed lines without red bands, the central value of the pre-LHC results (also discarding the LEP constraint).

One can see that in the CMSSM, VCMSSM and mSUGRA the heavier preferred spectra of the post-2010-LHC fits result in somewhat higher best-fit predictions for $M_{h}$. Only in the NUHM1, where the minimum was very shallow pre-LHC, does the best-fit value come out slightly lower. Now all four models predict, excluding the LEP constraint, best-fit values for $M_{h}$ above the SM LEP limit of $114.4 \mathrm{GeV}$ [58,59. One other significant effect of the 2010 LHC data on the oneparameter $\chi^{2}$ function in the NUHM1 is seen in the region $M_{h}<110 \mathrm{GeV}$. We recall that in the NUHM1 the LEP constraint is weakened at low $M_{h}$ because the $Z-Z-h$ coupling may be reduced: the 2010 LHC data help to close this loophole. Now most of the preferred $M_{h}$ region in the 

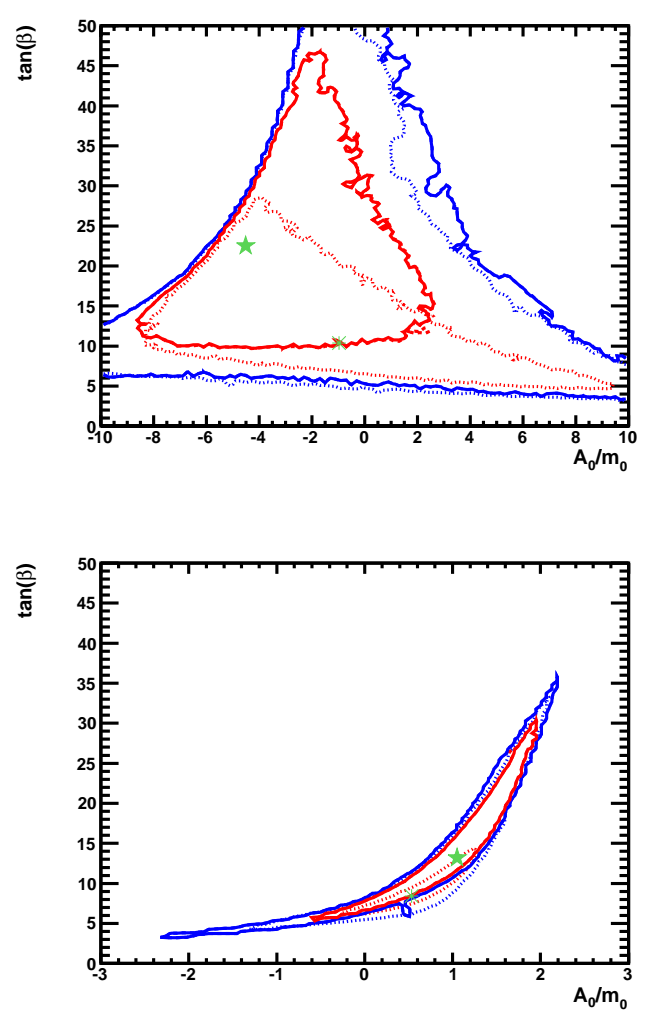
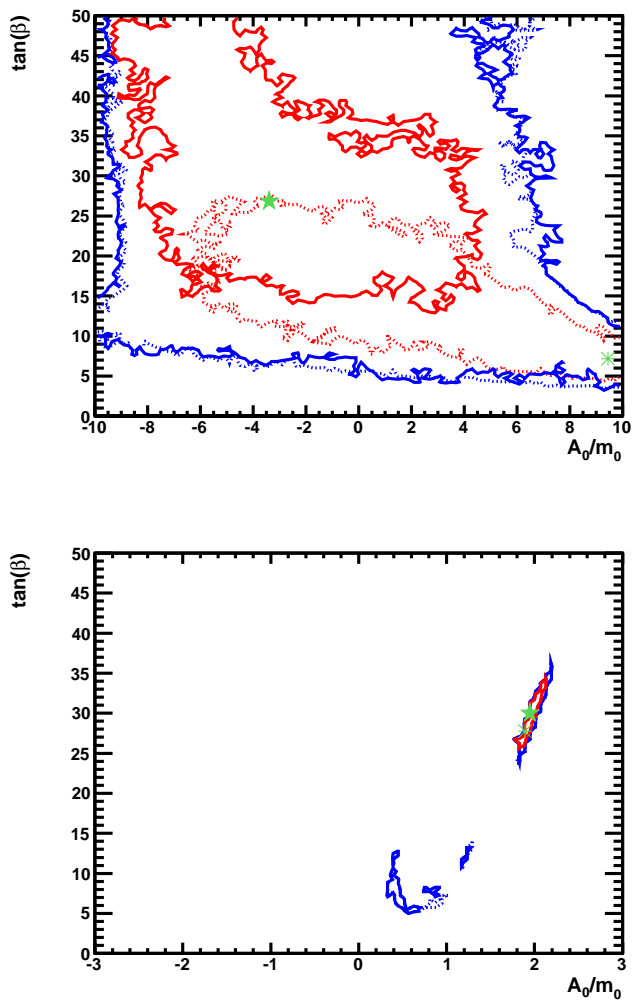

Figure 6. The $\left(A_{0} / m_{0}, \tan \beta\right)$ planes in the CMSSM (upper left panel), in the NUHM1 (upper right panel), in the VCMSSM (lower left panel) and in MSUGRA (lower right panel). In each plane, the best-fit point after incorporation of the 2010 LHC and Xenon100 constraints is indicated by a filled green star, and the pre-LHC fit by an open star. The 68 and 95\% CL regions are indicated by red and blue contours, respectively, the solid lines including the 2010 LHC and Xenon100 data, and the dotted lines including only the pre- $L H C$ data.

NUHM1 is indeed above $\sim 114 \mathrm{GeV}$, a tendency that was visible already in [2].

\section{Spin-independent dark matter scattering}

As a preface to discussing the importance of the uncertainties in the hadronic matrix elements used in the calculation of $\sigma_{p}^{\mathrm{SI}}$, we first display results that ignore these uncertainties. In Fig. 10, we show our previous pre-LHC, preXenon100 results in the $\left(m_{\tilde{\chi}_{1}^{0}}, \sigma_{p}^{\mathrm{SI}}\right)$ plane assuming $\Sigma_{\pi N}=50 \mathrm{MeV}$ as dotted curves, and postLHC but still pre-Xenon100 results (again assuming $\Sigma_{\pi N}=50 \mathrm{MeV}$ ) as dashed curves (red for
68\% CLs and blue for 95\% CLs), as calculated using SSARD 44. We also show the corresponding predictions with the higher value $\Sigma_{\pi N}=64 \mathrm{MeV}$ as duller coloured curves. The current Xenon100 results were not used in making these predictions, and we display separately the $95 \%$ CL limit on the cross section as a function of $m_{\tilde{\chi}_{1}^{0}}$ as well as the sensitivity bands from [24]. We see three important effects in these plots. One is that the 2010 LHC results push the predicted region in the $\left(m_{\tilde{\chi}_{1}^{0}}, \sigma_{p}^{\mathrm{SI}}\right)$ plane to higher masses, but not to very much lower values of $\sigma_{p}^{\text {SI }}$. The second effect is that the new Xenon100 constraint intersects the 

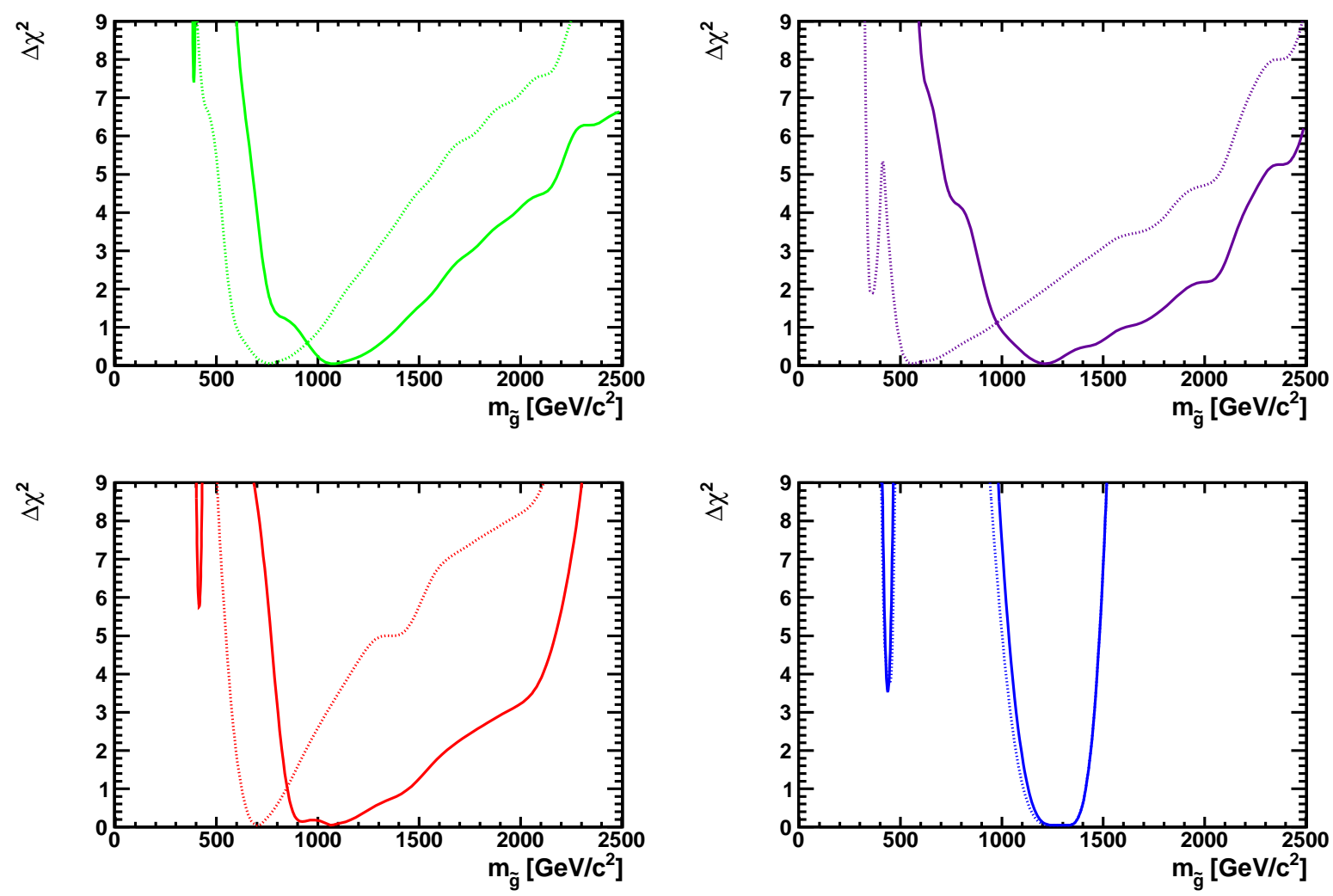

Figure 7. The $\chi^{2}$ likelihood functions of $m_{\tilde{g}}$ in the CMSSM (upper left), the NUHM1 (upper right), the VCMSSM (lower left) and $m S U G R A$ (lower right). The dashed curves are derived from the pre-LHC data set, and the solid curves include all the 2010 LHC constraints. In each case the value of $\Delta \chi^{2}$ relative to the respective best-fit point is displayed.

regions favoured in our pre- and post-2010-LHC analyses of the CMSSM and NUHM1. The third effect is that of the value of $\Sigma_{\pi N}$, which changes the predicted range of $\sigma_{p}^{\mathrm{SI}}$ by a factor $\sim 3$. The combination of these two latter effects means that any combination of accelerator and Xenon100 results must take careful account of the uncertainty in $\Sigma_{\pi N}$.

We now discuss the combination of the LHC and Xenon100 constraints in the $\left(m_{\tilde{\chi}_{1}^{0}}, \sigma_{p}^{\mathrm{SI}}\right)$ planes when the uncertainties in the hadronic matrix element $\Sigma_{\pi N}$ are included, as shown in Fig. 11 As usual, the dotted lines are preLHC and Xenon100, the dashed lines are post2010-LHC but pre-Xenon100, and the solid lines incorporate also the Xenon100 constraint, with our default assumption $\Sigma_{\pi N}=50 \pm 14 \mathrm{MeV} 16$. In the absence of the Xenon100 constraint, the LHC would have allowed values of $\sigma_{p}^{\mathrm{SI}}$ as large as $\sim 10^{-43} \mathrm{~cm}^{2}$ at the $95 \% \mathrm{CL}$ in the CMSSM and NUHM1, as seen in Fig. 10, whereas only values below $\sim 10^{-44} \mathrm{~cm}^{2}$ would have been expected

${ }^{16}$ These planes cannot be compared directly to those in 2 8, because here we use the SSARD code 44 to evaluate $\sigma_{p}^{\mathrm{SI}}$. This allows a more complete treatment of different contributions to the scattering rates than does MicrOMEGAs, including important uncertainties in the hadron scattering matrix elements 52. These lead, in particular, to larger ranges of $\sigma_{p}^{\text {SI }}$ for fixed values of $m_{\tilde{\chi}_{1}^{0}}$. We note in passing that MicrOMEGAs 40] uses $\Sigma_{\pi N}=55 \mathrm{MeV}$ as a default. 

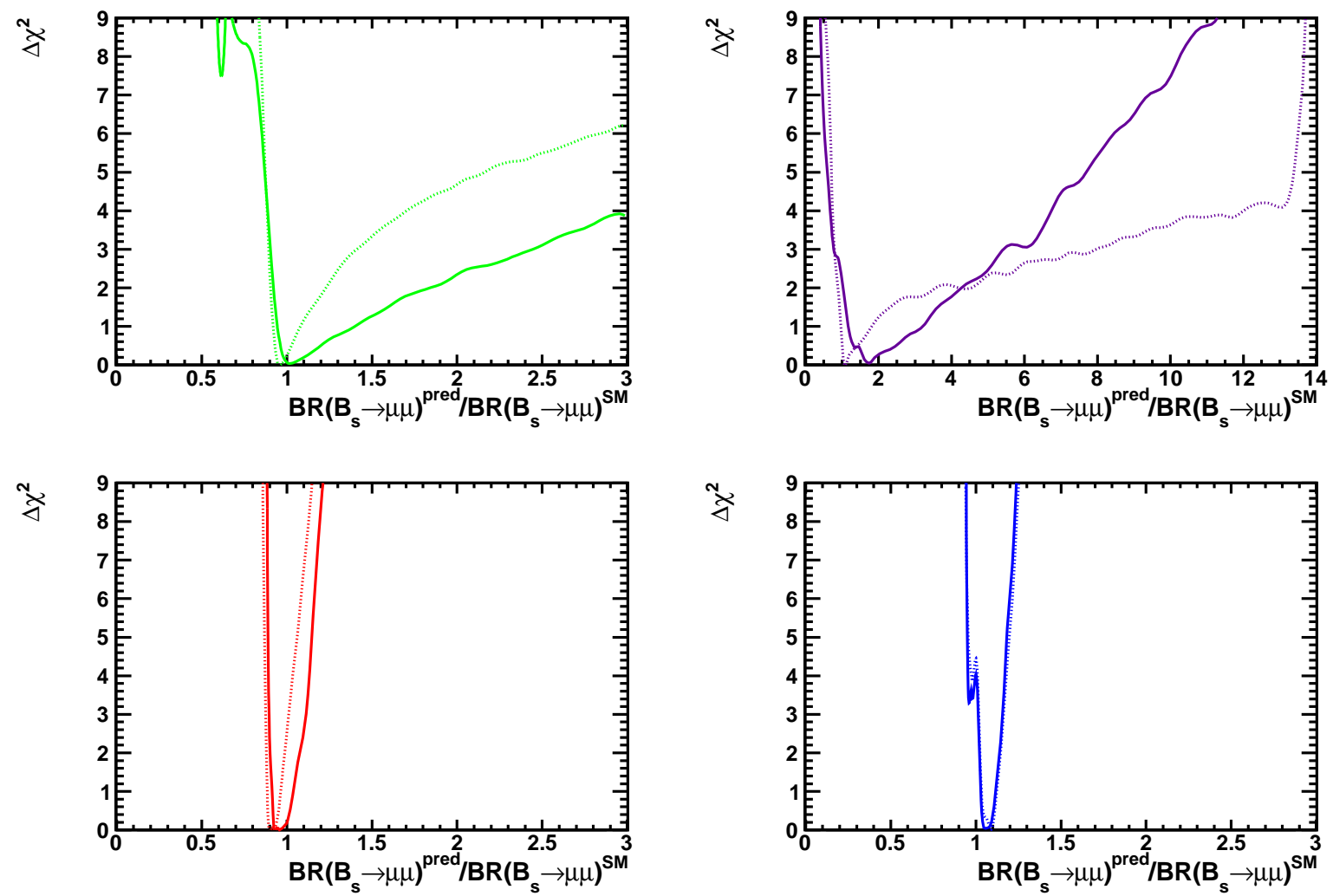

Figure 8. The $\chi^{2}$ likelihood functions of $\mathrm{BR}\left(B_{s} \rightarrow \mu^{+} \mu^{-}\right)$relative to the SM prediction in the CMSSM (upper left), the NUHM1 (upper right), the VCMSSM (lower left) and mSUGRA (lower right). The dashed curves are derived from the pre-LHC data set, and the solid curves include all the $2010 \mathrm{LHC}$ constraints. In each case the value of $\Delta \chi^{2}$ relative to the respective best-fit point is displayed.

at the $95 \%$ CL in the VCMSSM and mSUGRA. Since Xenon100 imposes $\sigma_{p}^{\text {SI }}<5 \times 10^{-44} \mathrm{~cm}^{2}$ for $m_{\tilde{\chi}_{1}^{0}} \sim 200 \mathrm{GeV}$, this constraint has significant impact in the CMSSM and NUHM1, as one could expect.

In Fig. 12 we compare our predictions for $\sigma_{p}^{\mathrm{SI}}$ after incorporation of the 2010 LHC data set and the Xenon100 constraint, for two different choices of $\Sigma_{\pi N}=50 \pm 14 \mathrm{MeV}$ (our default choice, shown in brighter colours) and $64 \pm 8 \mathrm{MeV}$ (a less conservative choice, shown in duller colours). As usual, the upper left panel shows predictions for the CMSSM, the upper right panel for is the NUHM1, the lower left panel shows the VCMSSM, and the lower right panel is for mSUGRA, and the $68 \%$
(95\%) CL regions are indicated by solid red (blue) contours. In all models, we find that the upper limits on $\sigma_{p}^{\text {SI }}$ are rather independent of the value assumed for $\Sigma_{\pi N}$. However, the lower bounds on $\sigma_{p}^{\text {SI }}$ are quite different for our default assumption $\Sigma_{\pi N}=50 \pm 14 \mathrm{MeV}$ and the comparison choice $\Sigma_{\pi N}=64 \pm 8 \mathrm{MeV}$, differing by a factor $\sim 3$. This means the interpretation of future direct dark matter search constraints will be hamstrung by this uncertainty.

\section{Spin-dependent dark matter scattering}

The SSARD code also provides as an output the spin-dependent LSP-proton cross section, $\sigma_{p}^{\mathrm{SD}}$, and we display in Fig. 13 the predictions for 

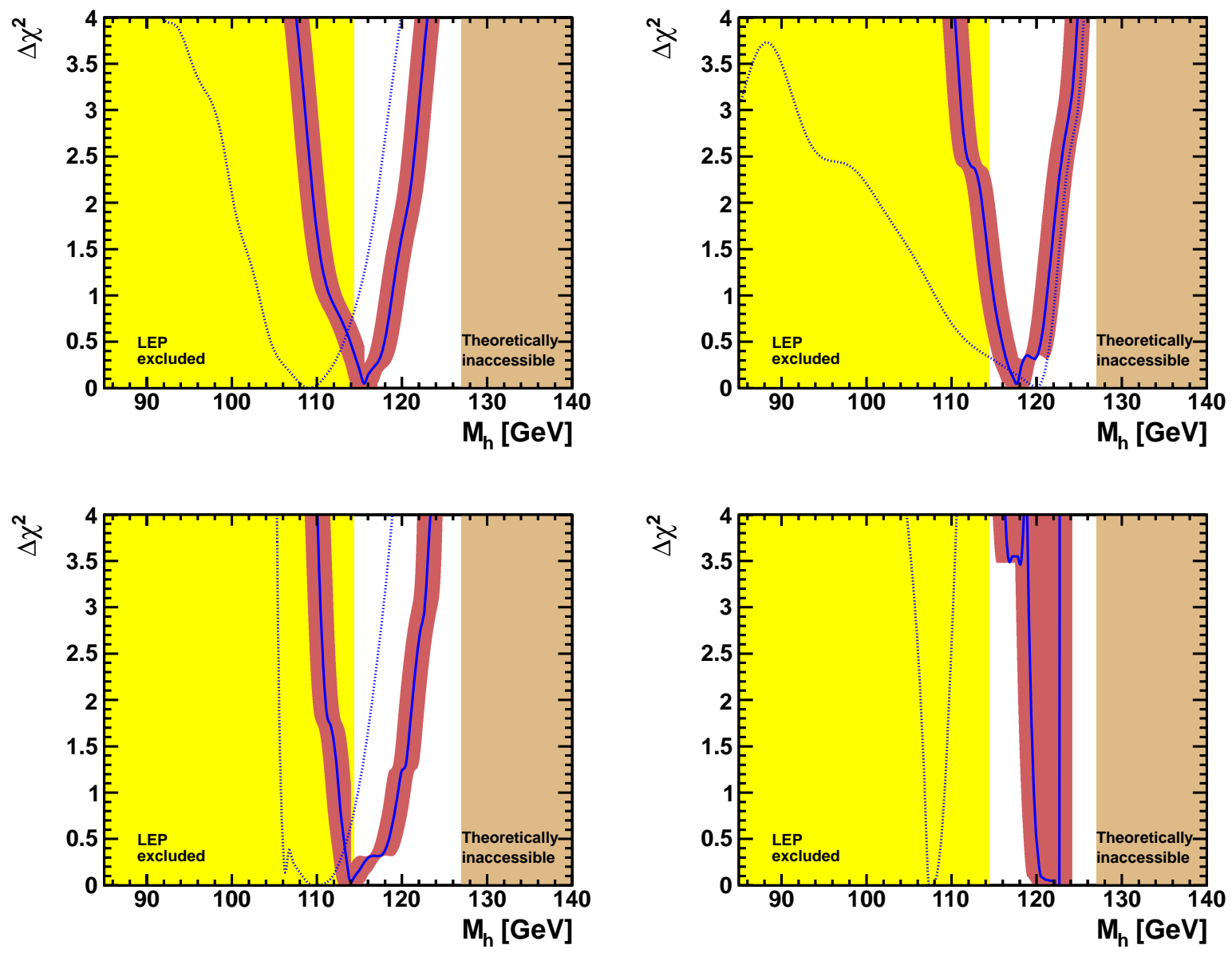

Figure 9. The one-parameter $\chi^{2}$ likelihood functions for the lightest MSSM Higgs mass $M_{h}$ in the CMSSM (upper left), NUHM1 (top right), VCMSSM (lower left) and mSUGRA (lower right). In each panel, we show the $\chi^{2}$ functions of the post-2010-LHC/Xenon100 constraints as solid lines, with a red band indicating the estimated theoretical uncertainty in the calculation of $M_{h}$ of $\sim 1.5 \mathrm{GeV}$, and the pre-LHC $\chi^{2}$ function is shown as a dashed line.

$\sigma_{p}^{\mathrm{SD}}$ from our likelihood analysis. We see that the range of $\sigma_{p}^{\mathrm{SD}}$ is much wider in the NUHM1 than in the other models, with both larger and smaller values being possible. Apart from the supersymmetric model parameters and the local galactic dark matter density, which we fix here to be $0.3 \mathrm{GeV} / \mathrm{cm}^{3}$, the principal uncertainty in calculating $\sigma_{p}^{\mathrm{SD}}$ is the hadronic spin-dependent scattering matrix element, which is dominated by the error in the strange axial-current matrix element, which we take to be $\left\langle N\left|\bar{s} \gamma_{\mu} s\right| N(s)\right\rangle=$ $-(0.09 \pm 0.03) \times s_{\mu}$, where $s_{\mu}$ is the nucleon spin vector. Proportionally, the uncertainty induced in $\sigma_{p}^{\mathrm{SD}}$ is far smaller than that induced in $\sigma_{p}^{\mathrm{SI}}$ 

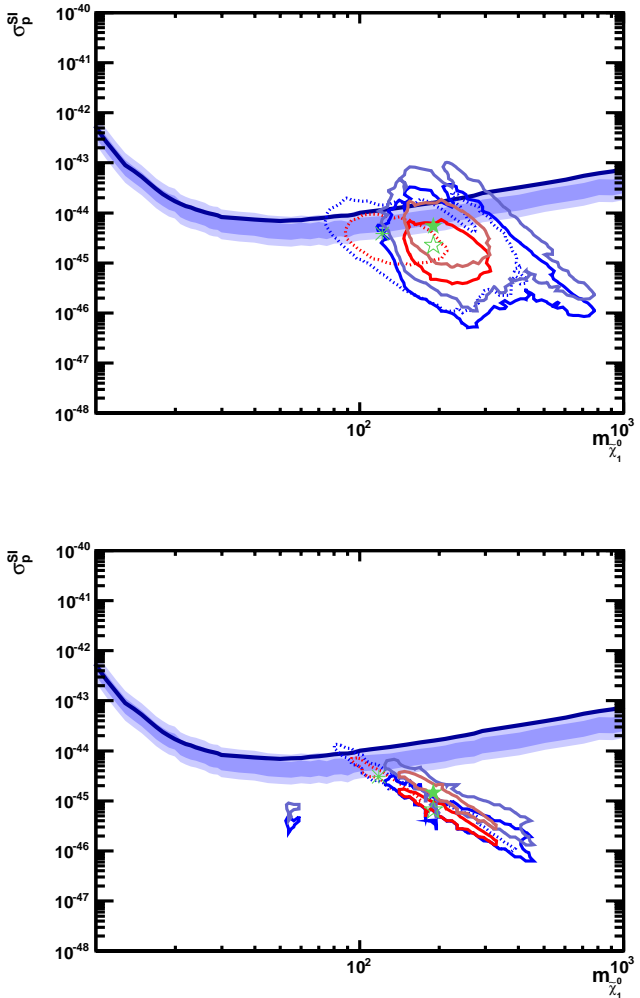
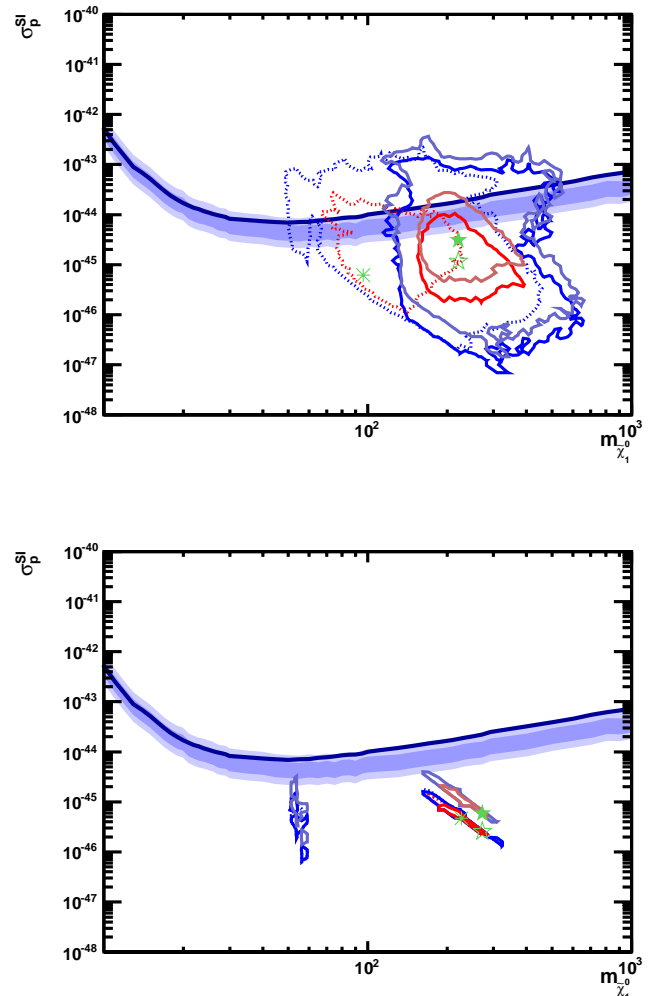

Figure 10. The correlation between the spin-independent dark matter scattering cross section $\sigma_{p}^{\mathrm{SI}}$ and $m_{\tilde{\chi}_{1}^{0}}$ prior to the inclusion of the current Xenon100 results in the CMSSM (upper left panel), in the NUHM1 (upper right panel), in the VCMSSM (lower left panel) and in $m S U G R A$ (lower right panel). In each panel, we show the 68 and 95\% CL contours (red and blue, respectively), the dotted curves correspond to our pre2010-LHC results, and the solid lines include the 2010 LHC results. Results assuming $\Sigma_{\pi N}=50 \mathrm{MeV}$ are shown as brighter coloured curves and $\Sigma_{\pi N}=64 \mathrm{MeV}$ as duller coloured curves, in each case disregarding uncertainties. The green 'snowflakes' (open stars) (filled stars) are the best-fit points in the corresponding models. Also shown is the 90\% CL Xenon100 upper limit [24] and its expected sensitivity band.

by the error in $\Sigma_{\pi N}$. As we see in Fig. 13, the most stringent direct experimental upper limit on $\sigma_{p}^{\mathrm{SD}}$ due to the COUPP Collaboration 60. (solid black line) lies above $10^{-38} \mathrm{~cm}^{2}$, significantly higher than our predictions in any of the CMSSM, NUHM1, VCMSSM and mSUGRA. More stringent upper limits on $\sigma_{p}^{\mathrm{SD}}$ are sometimes quoted based on experimental upper limits on energetic solar neutrinos that could be generated by LSP annihilations inside the sun 61]. These upper limits often assume that the LSPs are mainly cap- tured by spin-dependent scattering, which is not the case in general, and are in equilibrium inside the sun, which is also not the case in general 62. They also make simplifying assumptions about the annihilation final states that are not in general valid in the specific models studied here. Even with these assumptions, the upper limits lie above the ranges we predict in the CMSSM, VCMSSM and mSUGRA and barely touch the NUHM1 range. Therefore, a direct confrontation of these models with data on energetic solar neu- 

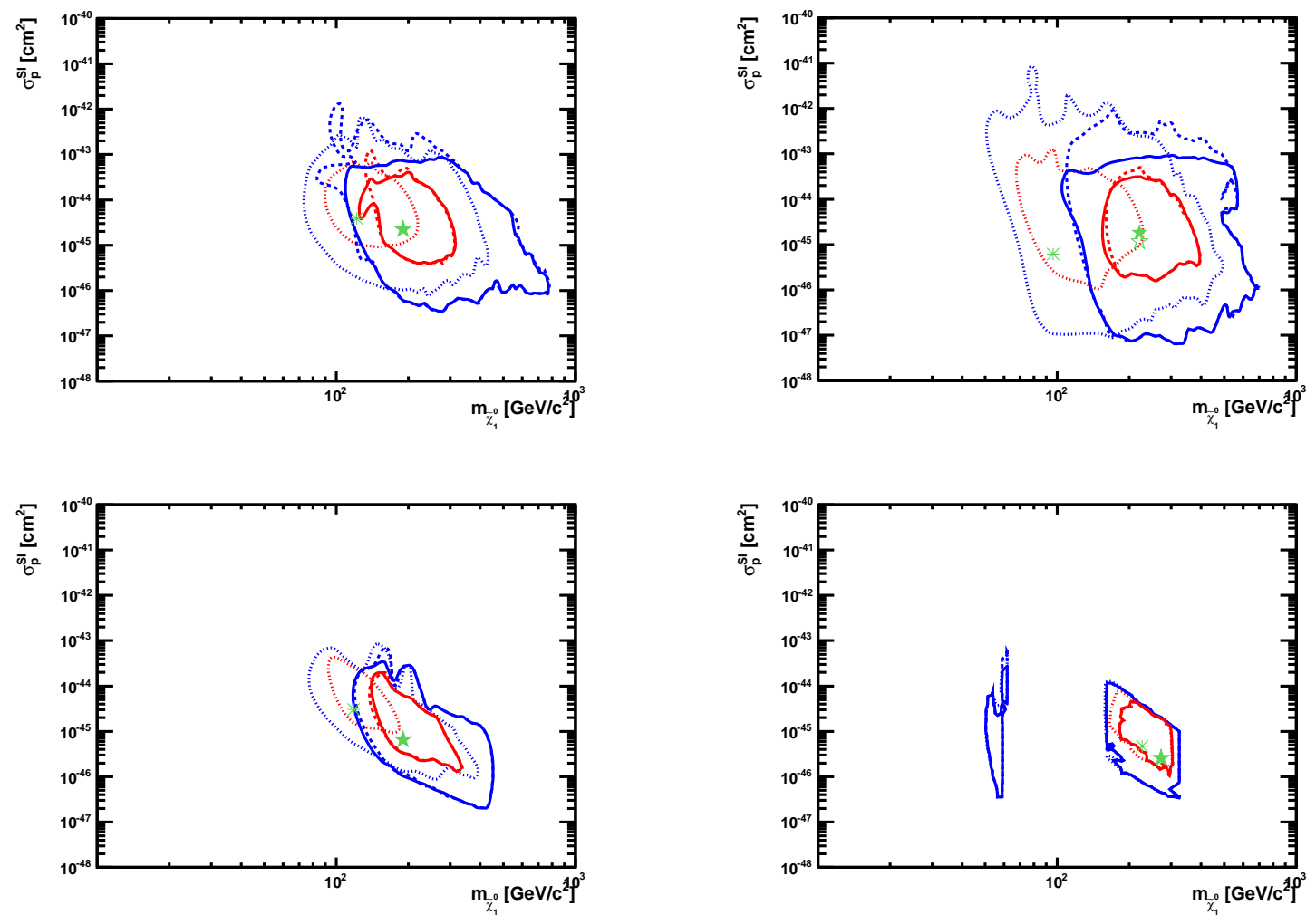

Figure 11. The correlation between $m_{\tilde{\chi}_{1}^{0}}$ and the spin-independent dark matter scattering cross section $\sigma_{p}^{\mathrm{SI}}$ calculated assuming a $\pi-N$ scattering $\sigma$ term $\Sigma_{\pi N}=50 \pm 14 \mathrm{MeV}$ in the CMSSM (upper left panel), in the NUHM1 (upper right panel), in the VCMSSM (lower left panel) and in mSUGRA (lower right panel). In each panel, we show as solid (dashed) lines the 68 and $95 \%$ CL contours (red and blue, respectively) after (before) applying the Xenon100 24 constraint. The green filled (open) stars are the best-fit points in each model including (excluding) the Xenon100 data. Also shown are best fit and 68 and $95 \%$ CL contours obtained from the pre-2010-LHC data set excluding the Xenon100 result (green 'snowflake' and dotted lines).

trinos still lies in the future.

\section{Summary and Discussion}

We have explored in this paper the implications of the 2010 LHC data for some of the simplest realizations of the MSSM, namely the CMSSM, the NUHM1, the VCMSSM and mSUGRA. In addition to the most sensitive available ATLAS and CMS searches for jets $+\mathbb{E}_{T}$, we have incorporated the constraints imposed by searches for the heavy MSSM Higgs bosons $H / A \rightarrow \tau^{+} \tau^{-}$and the constraints imposed by $\mathrm{LHCb}, \mathrm{CDF}$ and $\mathrm{D} \varnothing$ on $\operatorname{BR}\left(B_{s} \rightarrow \mu^{+} \mu^{-}\right)$, and we have also explored the impact of the direct Xenon100 search for dark matter scattering.

We have found that the ATLAS 0L and CMS MHT analyses shift the preferred regions in the $\left(m_{0}, m_{1 / 2}\right)$ planes as compared to the situation based on the initial CMS $\alpha_{T}$ and ATLAS 1L searches by amounts similar to those observed when comparing the results incorporating the 

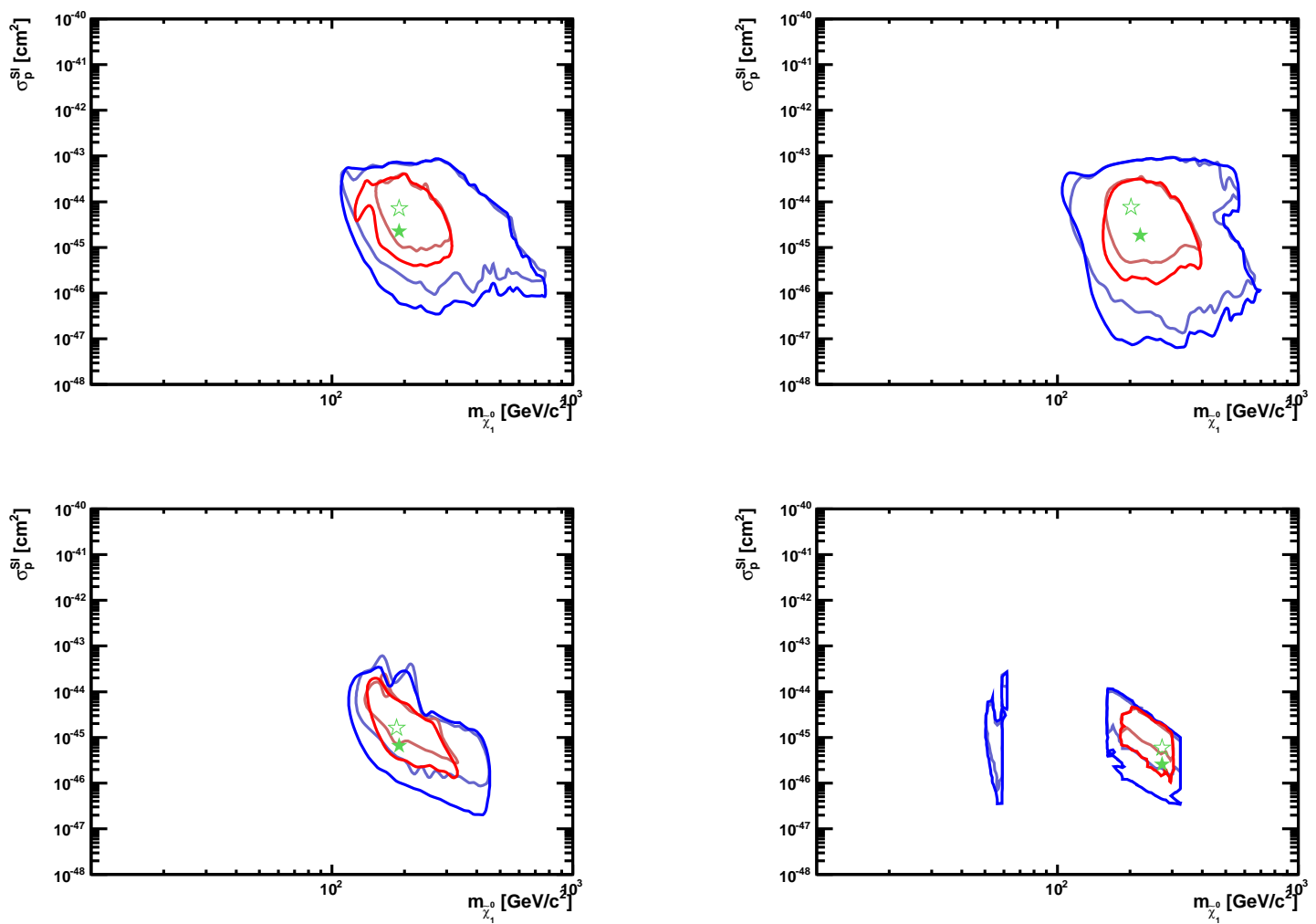

Figure 12. The correlation between the spin-independent dark matter scattering cross section $\sigma_{p}^{\mathrm{SI}}$ and $m_{\tilde{\chi}_{1}^{0}}$ after including the current Xenon100 results in the CMSSM (upper left panel), in the NUHM1 (upper right panel), in the VCMSSM (lower left panel) and in mSUGRA (lower right panel). In each panel, we show the 68 and $95 \%$ CL contours as solid red and blue lines, respectively. Results assuming $\Sigma_{\pi N}=50 \pm 14 \mathrm{MeV}$ are shown as brighter coloured curves, and those for $\Sigma_{\pi N}=64 \pm 8 \mathrm{MeV}$ are shown as duller coloured curves. The green filled (open) stars are the best-fit points in each case.

initial CMS $\alpha_{T}$ and ATLAS 1L searches with the pre-LHC situation. As a consequence, the preferred value of $m_{\tilde{g}}$ has been shifted upwards to $1 \mathrm{TeV}$ and beyond in the CMSSM, NUHM1 and VCMSSM. On the other hand, the picture in mSUGRA is not changed significantly by the newer ATLAS and CMS searches.

The CMS limits on heavy Higgs production and our compilation of LHCb, CDF and DØ constraints on $\operatorname{BR}\left(B_{s} \rightarrow \mu^{+} \mu^{-}\right)$have impacts on the parameter spaces of the NUHM1, but do not affect significantly the favoured regions of the CMSSM, VCMSSM and mSUGRA.
The Xenon100 results have an impact on the model parameter spaces that would be significant if $\Sigma_{\pi N}$ were large, $\sim 60 \mathrm{MeV}$. However, the current uncertainty in $\Sigma_{\pi N}$ does not permit a strong conclusion to be drawn, and we emphasize again the importance of experimental and theoretical attempts to reduce this uncertainty.

The adventure of the LHC search for SUSY has only just begun in 2010. The negative results of the searches to date are not in serious tension with the ranges of parameter spaces favoured preLHC in the models we have studied. The favoured regions yet to be explored offer good prospects 

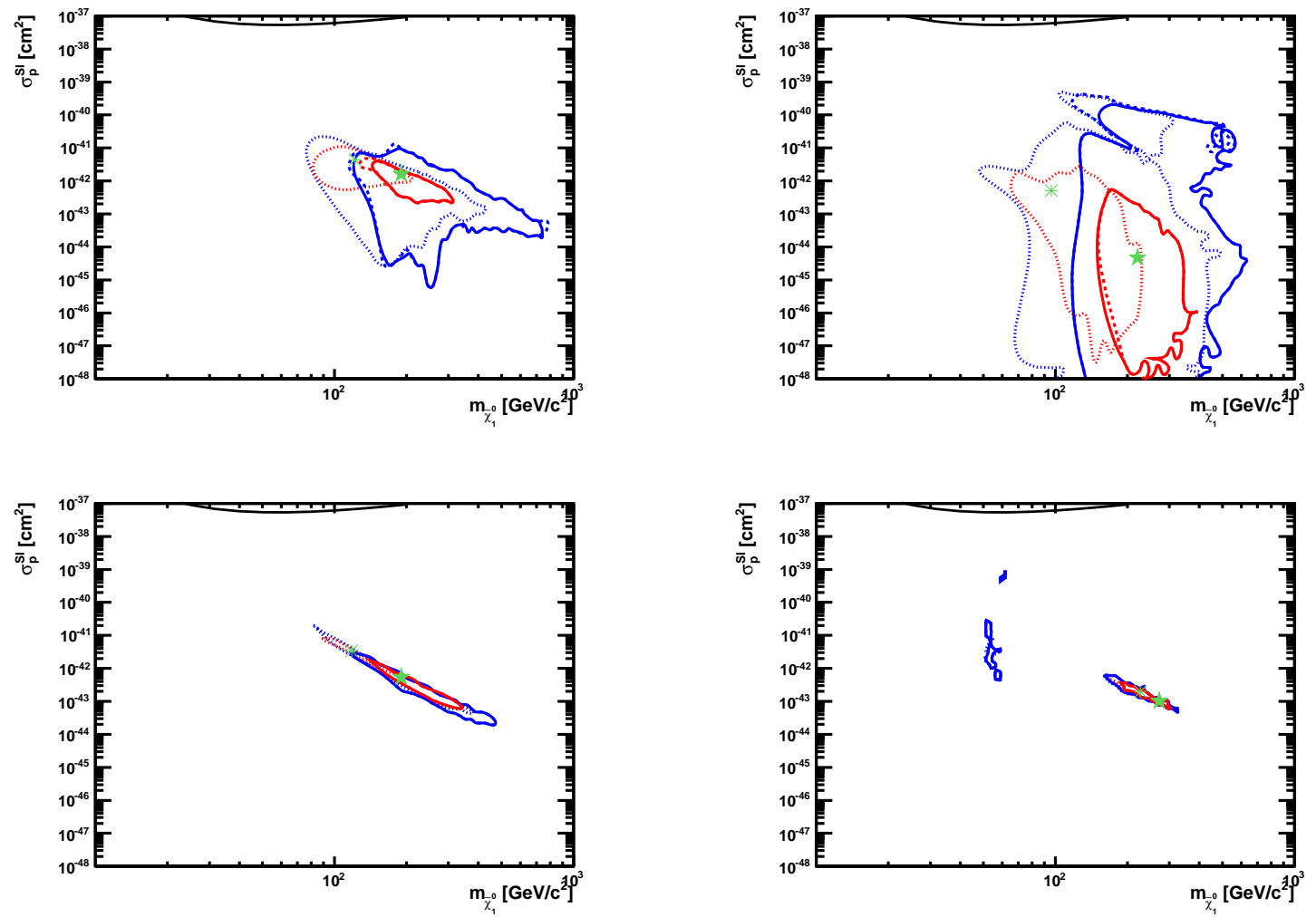

Figure 13. The correlation between the spin-dependent dark matter scattering cross section $\sigma_{p}^{\mathrm{SD}}$ (calculated assuming $\left\langle N\left|\bar{s} \gamma_{\mu} s\right| N(s)\right\rangle=-(0.09 \pm 0.03) \times s_{\mu}$, where $s_{\mu}$ is the nucleon spin vector) and $m_{\tilde{\chi}_{1}^{0}}$ in the CMSSM (upper left panel), in the NUHM1 (upper right panel), in the VCMSSM (lower left panel) and in mSUGRA (lower right panel). In each panel, we show the 68 and $95 \%$ CL contours (red and blue, respectively) including 2010 LHC data before and after applying the Xenon100 24 constraint (solid and dashed lines, respectively). The green filled (open) stars are the best-fit points obtained with these data sets. Also shown are best fit and the 68 and 95\% CL contours obtained from fits to the pre-2010-LHC data set excluding the Xenon100 result ('snowflake', dotted lines). We also show in each panel (solid black line) the 90\% CL upper limit on $\sigma_{p}^{\mathrm{SD}}$ provided by the COUPP Collaboration [60].

for the SUSY searches during the LHC run in $2011 / 12$. However, it is worthwhile to consider whether the exclusion by the LHC of very light squark and gluino masses may already have messages for future experimental studies of supersymmetry (if it exists).

There is much discussion about the possible next large collider project to follow the LHC, with high-energy lepton colliders among the favourites. A key question is the centre-of-mass energy of such a collider, and indications from the LHC are eagerly awaited. Any definitive statement on the impact of LHC results must surely wait at least until the end of the 2011/12 LHC run, and will require analyses that are less modelspecific than the results presented up to now. In this respect it has to be kept in mind that the LHC searches are mainly sensitive to the production of coloured particles, whereas lepton colliders will have a high sensitivity in particu- 
lar for the production of colour-neutral states, such as sleptons, charginos and neutralinos (and high-precision measurements furthermore provide an indirect sensitivity to quantum effects of new states). In this sense anything inferred from the coloured sector on the uncoloured sector depends on the underlying model assumptions, and in particular on assumptions about a possible universality of soft SUSY-breaking at the GUT scale.

The upward shifts for the preferred values of $m_{1 / 2}$ and, to a lesser extent, $m_{0}$, that we have found in the CMSSM, NUHM1 and VCMSSM upon inclusion of the 2010 LHC and Xenon100 constraints translate within those models into corresponding shifts in the production thresholds of supersymmetric particles at $e^{+} e^{-}$colliders. It has to be noted, however, that together with this upward shift of the preferred mass values we observe a significant decrease in the fit probabilities of those simple models, see Table 1. This indicates a slight tension in those models between the preference for rather light colour-neutral states arising in particular from $(g-2)_{\mu}$ and the search limits from the direct searches for coloured SUSY particles at the LHC. The mSUGRA scenario yields a significantly worse description of the data than the other considered models already for the pre-LHC data set, and inclusion of the 2010 LHC and Xenon100 constraints has only a small impact on the preferred fit values and the fit probabilities. If the upcoming LHC results lead to a further increase of the excluded mass regions for coloured superpartners, the CMSSM, NUHM1 and VCMSSM scenarios could eventually get under pressure. Such a tension could be avoided in realisations of SUSY with a larger splitting between the coloured and the colour-neutral part of the spectrum (for instance in GMSB-type scenarios), such that the masses of squarks and gluinos are in the $\mathrm{TeV}$ range, while sleptons, neutralinos and charginos can still be light.

Alternatively, the spectrum could be compressed, decreasing the splitting between the coloured and colour-neutral sparticles, leading to softer jets from gluino and squark decays, and hence less stringent constraints form searches for jets + missing transverse energy at the LHC [63].

\section{Noted Added}

The analysis of this paper provides a baseline with which 2011 LHC data can be confronted. While completing this work, we became aware of preliminary results from an analysis of events with $\geq 2$ jets, missing transverse energy and no detected leptons obtained with 165/pb of 2011 ATLAS data 64. In Fig. 14 we superpose on the $\left(m_{0}, m_{1 / 2}\right)$ planes shown previously in Fig. [1 the preliminary $95 \%$ CL limits obtained using a PCL approach (solid black line) and a $\mathrm{CL}_{s}$ approach (dash-dotted black line). We see that in the CMSSM (upper left) and VCMSSM (lower left) the new preliminary PCL 95\% contour runs very close to the best-fit point we find with the combined 2010 LHC data. It runs somewhat further away from the NUHM1 best-fit point, and outside our $68 \%$ CL contour for mSUGRA. The preliminary $\mathrm{CL}_{s}$ contour runs further below our best-fit points, still through our 68\% CL regions for the CMSSM, NUHM1 and VCMSSM but below our $68 \%$ CL region for mSUGRA. We defer incorporating this result into our analysis until a final version is published that enables its contribution to the global likelihood function to be modelled. However, this preliminary result already highlights the potential of the 2011 LHC run to probe deeper into supersymmetric parameter space.

\section{Acknowledgements}

The work of O.B., M.J.D. and J.E. is supported partly by the London Centre for Terauniverse Studies (LCTS), using funding from the European Research Council via the Advanced Investigator Grant 267352. The work of S.H. was supported in part by CICYT (grant FPA 2010-22163-C02-01) and by the Spanish MICINN's Consolider-Ingenio 2010 Program under grant MultiDark CSD2009-00064. The work of K.A.O. was supported in part by DOE grant DE-FG02-94ER-40823 at the University of Minnesota. K.A.O. also thanks SLAC (supported by the DOE under contract number DE-AC0276SF00515) and the Stanford Institute for Theoretical Physics for their hospitality and support. M.J.D. thanks CERN for hospitality during the 

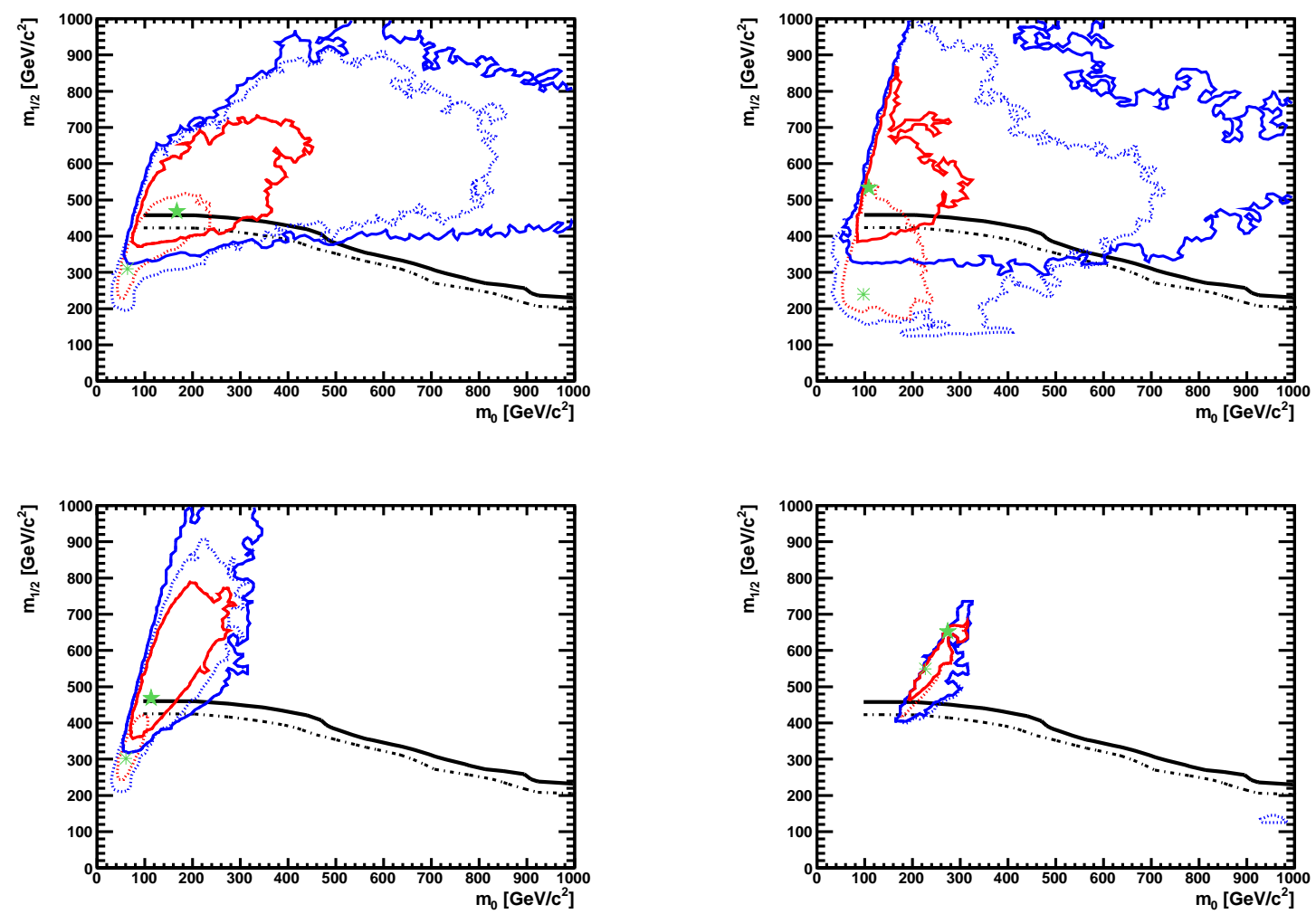

Figure 14. The $\left(m_{0}, m_{1 / 2}\right)$ planes in the CMSSM (upper left), the NUHM1 (upper right), the VCMSSM (lower left) and mSUGRA (lower right) as shown in Fig 1, now superposed by the preliminary $95 \%$ CL limits obtained by the ATLAS Collaboration 64 using a PCL approach (solid black lines) and a CL approach (dash-dotted black lines).

completion of this work.

\section{REFERENCES}

1. H. E. Haber and G. L. Kane, Phys. Rept. 117 (1985) 75 .

2. O. Buchmueller et al., Eur. Phys. J. C 71 (2011) 1634 [arXiv:1102.4585 [hep-ph]].

3. M. Drees and M. M. Nojiri, Phys. Rev. D 47 (1993) 376 arXiv:hep-ph/9207234; H. Baer and M. Brhlik, Phys. Rev. D 53 (1996) 597 arXiv:hep-ph/9508321; Phys. Rev. D 57 (1998) 567 |arXiv:hep-ph/9706509];

J. R. Ellis, T. Falk, K. A. Olive and M. Schmitt, Phys. Lett. B 388 (1996)
97 arXiv:hep-ph/9607292; Phys. Lett. B 413 (1997) 355 arXiv:hep-ph/9705444]; J. R. Ellis, T. Falk, G. Ganis, K. A. Olive and M. Schmitt, Phys. Rev. D 58 (1998) 095002 arXiv:hep-ph/9801445; V. D. Barger and C. Kao, Phys. Rev. D 57 (1998) 3131 arXiv:hep-ph/9704403; J. R. Ellis, T. Falk, G. Ganis and K. A. Olive, Phys. Rev. D 62 (2000) 075010 arXiv:hep-ph/0004169; J. R. Ellis, T. Falk, G. Ganis, K. A. Olive and M. Srednicki, Phys. Lett. B 510 (2001) 236 arXiv:hep-ph/0102098; V. D. Barger and C. Kao, Phys. Lett. B 518 (2001) 117 arXiv:hep-ph/0106189; L. Roszkowski, R. Ruiz de Austri and T. Nihei, JHEP 
0108 (2001) 024 arXiv:hep-ph/0106334; A. Djouadi, M. Drees and J. L. Kneur, JHEP 0108 (2001) 055 arXiv:hep-ph/0107316; U. Chattopadhyay, A. Corsetti and P. Nath, Phys. Rev. D 66 (2002) 035003 arXiv:hep-ph/0201001; J. R. Ellis, K. A. Olive and Y. Santoso, New Jour. Phys. 4 (2002) 32 arXiv:hep-ph/0202110; H. Baer, C. Balazs, A. Belyaev, J. K. Mizukoshi, X. Tata and Y. Wang, JHEP 0207 (2002) 050 arXiv:hep-ph/0205325; R. Arnowitt and B. Dutta, arXiv:hep-ph/0211417.

4. O. Buchmueller et al., Phys. Lett. B 657 (2007) 87 arXiv:0707.3447 [hep-ph]].

5. H. Baer, A. Mustafayev, S. Profumo, A. Belyaev and X. Tata, Phys. Rev. D 71 (2005) 095008 arXiv:hep-ph/0412059; H. Baer, A. Mustafayev, S. Profumo, A. Belyaev and X. Tata, JHEP 0507 (2005) 065, hep-ph/0504001, J. R. Ellis, K. A. Olive and P. Sandick, Phys. Rev. D 78 (2008) 075012 arXiv:0805.2343 [hep-ph]].

6. O. Buchmueller et al., JHEP 0809 (2008) 117 arXiv:0808.4128 [hep-ph]].

7. O. Buchmueller et al., Eur. Phys. J. C 64, 391 (2009) arXiv:0907.5568 [hep-ph]].

8. O. Buchmueller et al., Eur. Phys. J. C 71 (2011) 1583 arXiv:1011.6118 [hep-ph]].

9. J. R. Ellis, K. A. Olive, Y. Santoso and V. C. Spanos, Phys. Lett. B 573 (2003) 162 arXiv:hep-ph/0305212, and Phys. Rev. D 70 (2004) 055005 [arXiv:hep-ph/0405110.

10. O. Buchmueller et al., Phys. Rev. D 81 (2010) 035009 arXiv:0912.1036 [hep-ph]].

11. V. Khachatryan et al., CMS Collaboration, arXiv:1101.1628 [hep-ex].

12. G. Aad et al. [ATLAS Collaboration], arXiv:1102.2357 [hep-ex].

13. D. Feldman, K. Freese, P. Nath, B. D. Nelson and G. Peim, arXiv:1102.2548 [hep-ph]; B. C. Allanach, arXiv:1102.3149 [hep-ph]; S. Scopel, S. Choi, N. Fornengo and A. Bottino, arXiv:1102.4033 [hep-ph]; P. Bechtle et al., arXiv:1102.4693 [hep-ph]. B. C. Allanach, T. J. Khoo, C. G. Lester and S. L. Williams, arXiv:1103.0969 [hep-ph]; S. Akula, N. Chen, D. Feldman, M. Liu, Z. Liu, P. Nath and G. Peim, arXiv:1103.1197 [hep-ph]; S. Akula,
D. Feldman, Z. Liu, P. Nath and G. Peim, arXiv:1103.5061 [hep-ph]. M. J. Dolan, D. Grellscheid, J. Jaeckel, V. V. Khoze and P. Richardson, arXiv:1104.0585 [hep-ph].

14. H. Goldberg, Phys. Rev. Lett. 50 (1983) 1419; J. Ellis, J. Hagelin, D. Nanopoulos, K. Olive and M. Srednicki, Nucl. Phys. B 238 (1984) 453.

15. G. Aad et al. [ATLAS Collaboration], arXiv:1102.5290 [hep-ex].

16. G. Aad et al. [ATLAS Collaboration], http: //cdsweb.cern.ch/record/1345745/ files/ATLAS-CONF-2011-064.pdf, https://atlas.web.cern.ch/Atlas/GROUPS/ PHYSICS/CONFNOTES/ATLAS-CONF-2011-064/.

17. G. Aad et al. [ATLAS Collaboration], arXiv:1103.4344 [hep-ex]; V. Khachatryan et al. [CMS Collaboration], https://twiki.cern.ch/twiki/bin/view/ CMSPublic/PhysicsResultsSUS10011.

18. V. Khachatryan et al. [CMS Collaboration], arXiv.org:1103.1348 [hep-ex], https://twiki.cern.ch/twiki/bin/view/ CMSPublic/PhysicsResultsSUS10004, ...10006, ...10008; G. Aad et al. [ATLAS Collaboration], arXiv:1103.4344, 1103.6208, 1103.6214 [hep-ex], http://cdsweb.cern.ch/record/1338568/ files/ATLAS-CONF-2011-039.pdf.

19. G. Aad et al. [ATLAS Collaboration], http: //cdsweb.cern.ch/record/1336757/ files/ATLAS-CONF-2011-024.pdf.

20. V. Khachatryan et al. [CMS Collaboration], arXiv:1104.1619 [hep-ex].

21. R. Aaij et al. [LHCb Collaboration], arXiv:1103.2465 [hep-ex].

22. T. Aaltonen et al. [CDF Collaboration], Phys. Rev. Lett. 100, 101802 (2008) arXiv:0712.1708 [hep-ex]]; see also http://www-cdf .fnal.gov/physics/new/ bottom/090813.blessed-Bsd2mumu// bsmumupub3.7fb_v01.pdf.

23. V. M. Abazov et al. [D0 Collaboration], Phys. Lett. B 693 (2010) 539 arXiv:1006.3469 [hep$\mathrm{ex}]$.

24. E. Aprile et al. [XENON100 Collaboration], arXiv:1104.2549 [astro-ph.CO].

25. S. Akula, D. Feldman, Z. Liu, P. Nath 
and G. Peim, arXiv:1103.5061 [hep-ph] (v2); M. Farina, M. Kadastik, D. Pappadopulo, J. Pata, M. Raidal and A. Strumia, arXiv:1104.3572 [hep-ph].

26. S. Profumo, arXiv:1105.5162 [hep-ph].

27. T. Li, J. A. Maxin, D. V. Nanopoulos and J. W. Walker, arXiv:1106.1165 [hep-ph].

28. See: http://cern.ch/mastercode .

29. B. C. Allanach, Comput. Phys. Commun. 143 (2002) 305 arXiv:hep-ph/0104145.

30. G. Degrassi, S. Heinemeyer, W. Hollik, P. Slavich and G. Weiglein, Eur. Phys. J. C 28 (2003) 133 arXiv:hep-ph/0212020.

31. S. Heinemeyer, W. Hollik and G. Weiglein, Eur. Phys. J. C 99 (1999) 343 arXiv:hep-ph/9812472.

32. S. Heinemeyer, W. Hollik and G. Weiglein, Comput. Phys. Commun. 124 (2000) 76 arXiv:hep-ph/9812320; See http://www.feynhiggs.de .

33. M. Frank et al., JHEP 0702 (2007) 047 arXiv:hep-ph/0611326.

34. G. Isidori and P. Paradisi, Phys. Lett. B 639 (2006) 499 arXiv:hep-ph/0605012.

35. G. Isidori, F. Mescia, P. Paradisi and D. Temes, Phys. Rev. D 75 (2007) 115019 arXiv:hep-ph/0703035, and references therein.

36. F. Mahmoudi, Comput. Phys. Commun. 178 (2008) 745 arXiv:0710.2067 [hep-ph]]; Comput. Phys. Commun. 180 (2009) 1579 arXiv:0808.3144 [hep-ph]].

37. D. Eriksson, F. Mahmoudi and O. Stal, JHEP 0811 (2008) 035 arXiv:0808.3551 [hep-ph]].

38. S. Heinemeyer et al., JHEP 0608 (2006) 052 arXiv:hep-ph/0604147.

39. S. Heinemeyer, W. Hollik, A. M. Weber and G. Weiglein, JHEP 0804 (2008) 039 arXiv:0710.2972 [hep-ph]].

40. G. Belanger, F. Boudjema, A. Pukhov and A. Semenov, Comput. Phys. Commun. 176 (2007) 367 arXiv:hep-ph/0607059; Comput. Phys. Commun. 149 (2002) 103 arXiv:hep-ph/0112278; Comput. Phys. Commun. $\mathbf{1 7 4}$ (2006) 577 arXiv:hep-ph/0405253.

41. P. Gondolo et al., New Astron. Rev. 49 (2005) 149; JCAP 0407 (2004) 008
arXiv:astro-ph/0406204

42. P. Skands et al., JHEP 0407 (2004) 036 arXiv:hep-ph/0311123.

43. B. Allanach et al., Comput. Phys. Commun. 180 (2009) 8 arXiv:0801.0045 [hep-ph]].

44. Information about this code is available from K. A. Olive: it contains important contributions from T. Falk, G. Ganis, A. Mustafayev, J. McDonald, K. A. Olive, P. Sandick, Y. Santoso and M. Srednicki.

45. V. Khachatryan et al. [CMS Collaboration], http://cdsweb.cern.ch/record/1342547/ files/SUS-11-001-pas.pdf.

46. G. Aad et al. [ATLAS Collaboration], https://ATLAS . web. cern. ch/ATLAS/GROUPS/ PHYSICS/PAPERS/susy-0lepton_01/.

47. V. Khachatryan et al. [CMS Collaboration], http://cdsweb.cern.ch/record/1343076/ files/SUS-10-005-pas.pdf; see also https://twiki.cern.ch/twiki/bin/view/ CMSPublic/PhysicsResultsSUS.

48. V. Khachatryan et al. [CMS Collaboration], https://twiki.cern.ch/twiki/bin/view/ CMSPublic/PhysicsResultsHIG10002/

49. R. V. Harlander, W. B. Kilgore, Phys. Rev. D68 (2003) 013001 arXiv:hep-ph/0304035.

50. The Heavy Flavor Averaging Group, D. Asner et al., arXiv:1010.1589 [hep-ex], with updates available at http://www.slac.stanford.edu/xorg/ $\mathrm{hfag} / \mathrm{osc} / \mathrm{end}$ 2009.

51. A. Bottino, F. Donato, N. Fornengo and S. Scopel, Astropart. Phys. 13 (2000) 215 arXiv:hep-ph/9909228; E. Accomando, R. L. Arnowitt, B. Dutta and Y. Santoso, Nucl. Phys. B $\mathbf{5 8 5}$ (2000) 124 arXiv:hep-ph/0001019; J. R. Ellis, K. A. Olive, Y. Santoso and V. C. Spanos, Phys. Rev. D 71 (2005) 095007 arXiv:hep-ph/0502001].

52. J. R. Ellis, K. A. Olive and C. Savage, Phys. Rev. D 77 (2008) 065026 arXiv:0801.3656 [hep-ph]].

53. J. Gasser, H. Leutwyler and M. E. Sainio, Phys. Lett. B 253 (1991) 252; M. Knecht, PiN Newslett. 15 (1999) 108 arXiv:hep-ph/9912443; M. E. Sainio, PiN Newslett. 16 (2002) 
138 arXiv:hep-ph/0110413; B. Borasoy and U. G. Meissner, Annals Phys. 254 (1997) 192 arXiv:hep-ph/9607432.

54. M. M. Pavan, I. I. Strakovsky, R. L. Workman and R. A. Arndt, PiN Newslett. 16 (2002) 110 arXiv:hep-ph/0111066.

55. M. M. Pavan, private communication (2011), taking into account recent data on pionic Deuterium: see T. Strauch et al., arXiv:1011.2415 [nucl-ex], as interpreted in V. Baru, C. Hanhart, M. Hoferichter, B. Kubis, A. Nogga and D. R. Phillips, arXiv:1003.4444 [nucl-th].

56. R. D. Young and A. W. Thomas, Phys. Rev. D 81 (2010) 014503 arXiv:0901.3310 [heplat]].

57. J. Giedt, A. W. Thomas and R. D. Young, Phys. Rev. Lett. 103 (2009) 201802 arXiv:0907.4177 [hep-ph]].

58. R. Barate et al. [ALEPH, DELPHI, L3, OPAL Collaborations and LEP Working Group for Higgs boson searches], Phys. Lett. B 565 (2003) 61 arXiv:hep-ex/0306033.

59. S. Schael et al. [ALEPH, DELPHI, L3, OPAL Collaborations and LEP Working Group for Higgs boson searches], Eur. Phys. J. C 47 (2006) 547 arXiv:hep-ex/0602042.

60. E. Behnke et al. [COUPP Collaboration], Phys. Rev. Lett. 106 (2011) 021303 arXiv:1008.3518 [astro-ph.CO]].

61. S. Desai et al. [SuperKamiokande Collaboration], Phys. Rev. D 70 (2004) 083523; R. Abbasi et al. [IceCube Collaboration], Phys. Rev. Lett. 102 (2009) 201302.

62. J. Ellis, K. A. Olive, C. Savage and V. C. Spanos, Phys. Rev. D 81 (2010) 085004 arXiv:0912.3137 [hep-ph]]; Phys. Rev. D 83 (2011) 085023 [arXiv:1102.1988 [hep-ph]].

63. T. J. LeCompte and S. P. Martin, arXiv:1105.4304 [hep-ph]; J. Fan, M. Reece and J. T. Ruderman, arXiv:1105.5135 [hep-ph].

64. G. Aad et al. [ATLAS Collaboration], http://cdsweb.cern.ch/record/1356194/ files/ATLAS-CONF-2011-086.pdf; see also https://atlas.web.cern.ch/Atlas/GROUPS/ PHYSICS/CONFNOTES/ATLAS-CONF-2011-086/. 